\title{
COMPACTNESS OF THE COMPLEX GREEN OPERATOR ON CR-MANIFOLDS OF HYPERSURFACE TYPE
}

\author{
ANDREW RAICH
}

\begin{abstract}
The purpose of this article is to study compactness of the complex Green operator on $\mathrm{CR}$ manifolds of hypersurface type. We introduce $\left(\mathrm{CR}-P_{q}\right)$, a potential theoretic condition on $(0, q)$-forms that generalizes Catlin's property $\left(P_{q}\right)$ to CR manifolds of arbitrary codimension. We prove that if an embedded CR-manifold of hypersurface type of real dimension at least five satisfies $\left(\mathrm{CR}-P_{q}\right)$ and $\left(\mathrm{CR}-P_{n-1-q}\right)$, then the complex Green operator is a compact operator on the Sobolev spaces $H_{0, q}^{s}(M)$ and $H_{0, n-1-q}^{s}(M)$, if $1 \leq q \leq n-2$ and $s \geq 0$. We use CR-plurisubharmonic functions to build a microlocal norm that controls the totally real direction of the tangent bundle.
\end{abstract}

\section{IntRoduction AND RESUlts}

In this article, we introduce property $\left(\mathrm{CR}-P_{q}\right)$, a potential theoretic condition on $(0, q)$ forms. We show that if an embedded CR-manifold of hypersurface type satisfies $\left(\mathrm{CR}-P_{q}\right)$ and $\left(\mathrm{CR}-P_{n-1-q}\right)$, then the complex Green operator is a compact operator on the Sobolev spaces $H_{0, q}^{s}(M)$ and $H_{0, n-1-q}^{s}(M)$ if $1 \leq q \leq n-2$. We use CR-plurisubharmonic functions to build a microlocal norm that controls the "bad" direction of the tangent bundle. We first prove the closed range and compactness results on $L_{0, q}^{2}(M)$ and use an elliptic regularization argument to pass to higher Sobolev spaces.

A CR-manifold of hypersurface type $M$ is the generalization to higher codimension of the boundary of a pseudoconvex domain. Let $\Omega \subset \mathbb{C}^{N}$ be a pseudoconvex domain and $H$ be a holomorphic function on the closure of $\Omega$. If $h$ is the boundary value of $H$, then $h$ satisfies the tangential Cauchy-Riemann equations $\bar{\partial}_{b} h=0$. As with the Cauchy-Riemann operator, $\bar{\partial}_{b}$ gives rise to a complex that is a useful tool for analyzing the behavior of forms on and near the boundary. A CR-manifold of hypersurface type is a $(2 n-1)$-dimensional manifold that is locally equivalent to a hypersurface in $\mathbb{C}^{n}$. The tangential Cauchy-Riemann operator $\bar{\partial}_{b}$ can again be thought of as the restriction of $\bar{\partial}$ to $M$.

The $L^{2}$-theory of $\bar{\partial}_{b}$ has been studied when $M$ is a CR-manifold of hypersurface type. When $M$ is the boundary of a pseudoconvex domain, it is by now classical that $\bar{\partial}_{b}$ has closed range [Koh86, Sha85b, BS86]. More recent work by Nicoara [Nic06] shows the same result holds when $M$ a CR-manifold of hypersurface type. The approach to analyze $\bar{\partial}_{b}$-problems proceeds down one of two paths. One is to follow Shaw's approach and use $\bar{\partial}$-techniques and jump formulas, and the other path is to use Kohn's ideas and develop a microlocal

2000 Mathematics Subject Classification. 32W10, (35N10, 32V20, 35A27). 
analysis to control the totally real or "bad" direction of the tangent bundle. When $M$ is not a hypersurface, microlocal analysis seems to be a more natural approach, and we will use this approach.

The method that we use to solve the $\bar{\partial}_{b}$-equation is to introduce the Kohn Laplacian $\square_{b}=\bar{\partial}_{b}^{*} \bar{\partial}_{b}+\bar{\partial}_{b} \bar{\partial}_{b}^{*}$ and invert it. The inverse (modulo its null space) is called the complex Green operator and denoted $G_{q}$ when it acts on $L_{0, q}^{2}(M)$, and the canonical solution to $\bar{\partial}_{b} u=f$ is given by $u=\bar{\partial}_{b}^{*} G_{q} f$ (assuming $f$ satisfies the appropriate compatibility condition, e.g., $\bar{\partial}_{b} f=0$ when $\left.1 \leq q \leq n-2\right)$. Closed range of $\bar{\partial}_{b}$ implies that $G_{q}$ exists and is bounded on $L^{2}$, though geometric and potential theoretic properties of $M$ can give $G_{q}$ much stronger regularity properties. These additional regularity properties, however, have only been explored when $M=\mathrm{b} \Omega$ is the boundary of a pseudoconvex domain. In this case, subellipticity of $G_{q}$ holds if and only if $M$ satisfies a curvature condition called finite type (at the symmetric level $q$ and $n-1-q$ ) [Cat83, Cat87, Koh02, Nic, Dia86, Koe04, RS08]. Optimal subelliptic estimates (so called maximal estimates) were obtained in [Koe02 under the additional condition that all eigenvalues of the Levi form are comparable. This work unifies earlier results for strictly pseudoconvex domains and for domains of finite type in $\mathbb{C}^{2}$. For general domains, it is known that if $\Omega$ admits a defining function that is plurisubharmonic at points of the boundary, then $G_{q}$ preserves the Sobolev spaces $H^{s}(\mathrm{~b} \Omega), s \geq 0$ [BS91]. A defining function is called plurisubharmonic at the boundary when its complex Hessian at points of the boundary is positive semidefinite in all directions. For example, all convex domains admit such defining functions.

On a pseudoconvex domain $\Omega \subset \mathbb{C}^{N}$, the $\bar{\partial}$-Neumann operator is the inverse to the $\bar{\partial}$ Neumann Laplacian $\square=\bar{\partial} \bar{\partial}^{*}+\bar{\partial}^{*} \bar{\partial}$ on $L_{0, q}^{2}(\Omega)$. When $q=1$, a necessary and sufficient condition for subellipticity of the $\bar{\partial}$-Neumann operator on $\Omega$ is the existence of a plurisubharmonic function whose complex Hessian blows up proportional to a reciprocal power of the distance to the boundary [Cat83, Cat87, Str97]. In [Cat84, Catlin introduces a weakened version of complex Hessian blowup condition and instead requires only that there exist plurisubharmonic functions with arbitrarily large complex Hessians. He calls this condition property $(\mathrm{P})$ and its natural generalization to $(0, q)$-forms, called $\left(P_{q}\right)$, is now a well known sufficient condition for compactness of the $\bar{\partial}$-Neumann operator (see [FS01, Str06] for a discussion of compactness in the $\bar{\partial}$-Neumann problem). In [RS08], Emil Straube and I show that if $M=\mathrm{b} \Omega$ is the boundary of a smooth, bounded, pseudoconvex domain and satisfies $\left(P_{q}\right)$ and $\left(P_{n-1-q}\right)$, then $G_{q}$ is a compact operator on $L_{0, q}^{2}(M)$. We also show that compactness of $G_{q}$ implies compactness of the $\bar{\partial}$-Neumann operator on $(0, q)$-forms on $\Omega$ and if $\mathrm{b} \Omega$ is locally convexifiable then $\left(P_{q}\right)$ and $\left(P_{n-1-q}\right)$ is equivalent to compactness of $G_{q}$ (see [FS98] as well). Our methods involve $\bar{\partial}$-techniques, a jump formula in the spirit of Shaw (and Boas) Sha85b, BS86, and a detailed study of compactness of the $\bar{\partial}$-Neumann operator on the annulus between two pseudoconvex domains. Applying $\bar{\partial}$-techniques to investigate the complex Green operator in the higher codimension case investigated in this article seems to be difficult if $q>1$ because it is unknown if $\left(P_{q}\right)$ is invariant under CR-equivalences (or even biholomorphisms that are not conformal mappings) if $q>1$. 
The goal of this article is to generalize the compactness result of [RS08] to the case when $M$ is a CR-manifold of hypersurface type. We introduce property $\left(\mathrm{CR}-P_{q}\right)$, a generalization of $\left(P_{q}\right)$ for CR-manifolds of hypersurface type, and show that it is a sufficient condition for compactness of the complex Green operator.

Let

$$
\mathcal{H}^{q}=\left\{\varphi \in L_{0, q}^{2}(M) \cap \operatorname{Dom}\left(\bar{\partial}_{b}\right) \cap \operatorname{Dom}\left(\bar{\partial}_{b}^{*}\right): \bar{\partial}_{b} \varphi=0, \bar{\partial}_{b}^{*} \varphi=0\right\}
$$

be the space of harmonic forms and

$$
{ }^{\perp} \mathcal{H}^{q}=\left\{\varphi \in L_{0, q}^{2}(M):(\varphi, \phi)_{0}=0, \text { for all } \phi \in \mathcal{H}\right\} .
$$

Our main result is the following theorem.

Theorem 1.1. Let $M \subset \mathbb{C}^{N}$ be a smooth, compact, orientable weakly pseudoconvex $C R$ manifold of hypersurface type of real dimension at least five that satisfies $\left(C R-P_{q}\right)$ and $(C R$ $\left.P_{n-1-q}\right)$. If $1 \leq q \leq n-2$ and $s \geq 0$, then

(i) $\bar{\partial}_{b}$ and $\bar{\partial}_{b}^{*}$ acting on $H_{0, q}^{s}(M)$ have closed range,

(ii) the complex Green operator $G_{q}$ exists and is a compact operator on $H_{0, q}^{s}(M)$,

(iii) $\mathcal{H}^{q}$ is finite dimensional.

The assumption that $1 \leq q \leq n-2$ excludes the endpoints $q=0$ and $q=n-1$. For the endpoint case, it is not clear what $\left(\mathrm{CR}-P_{0}\right)$ should be. However, one can check (in analogy to the $\bar{\partial}$-Neumann problem) that $G_{0}=\bar{\partial}_{b}^{*} G_{1}^{2} \bar{\partial}_{b}=\bar{\partial}_{b}^{*} G_{1}\left(\bar{\partial}_{b}^{*} G_{1}\right)^{*}$, and thus it follows that (CR- $\left.P_{1}\right)$ is a sufficient condition for compactness of $G_{0}$ (and $G_{n-1}$ as well). The requirement that the dimension of $M$ is at least five is a seemingly technical assumption concerning the eigenvalues of a Hermitian matrix. In particular, and $H=\left(h_{j k}\right)$ is a Hermitian, positive definite matrix, $1 \leq i, k \leq n-1$, then $\left(\delta_{j k} \sum_{\ell=1}^{n-1} h_{\ell \ell}-h_{j k}\right)$ is a Hermitian, positive definite matrix if $n \geq 3$. This fact is false when $n=2$, and this causes the three dimensional case to remain open.

The symmetric requirements at level $q$ and $n-1-q$ are necessary [Koe04, RS08, Koh81]. To a $(0, q)$-form $u$ on $\mathrm{b} \Omega$, there is an associated $(0, n-1-q)$-form $\tilde{u}$ (obtained through a modified Hodge-* construction) such that $\|u\| \approx\|\tilde{u}\|, \bar{\partial}_{b} \tilde{u}=(-1)^{q} \widetilde{\left(\bar{\partial}_{b}^{*} u\right)}$, and $\bar{\partial}_{b}^{*} \tilde{u}=(-1)^{q+1} \widetilde{\left(\overline{\bar{\partial}}_{b} u\right)}$, modulo terms that are $O(\|u\|)$. Consequently, a compactness estimate holds for $(0, q)$-forms if and only if the corresponding estimate holds for $(0, n-1-q)$-forms. In view of the characterization of compactness on convex domains [FS98], such a symmetry between form levels is absent in the $\bar{\partial}$-Neumann problem. (The analogous construction performed for forms on $\Omega$ yields a form $\tilde{u}$ that in general is not in the domain of $\bar{\partial}^{*}$.)

A consequence of Theorem 1.1 and Corollary 3.3 is the following generalization of Theorem 1.4 in [RS08].

Corollary 1.2. Let $M \subset \mathbb{C}^{N}$ be a smooth, compact, orientable weakly pseudoconvex $C R$ manifold of hypersurface type that satisfies $\left(P_{q}\right)$. Then $M$ satisfies $\left(C R-P_{q}\right)$. In particular, if $M$ satisfies $\left(P_{q}\right)$ and $\left(P_{n-1-q}\right)$ and is of real dimension at least five, then the conclusions of Theorem 1.1 hold. 
I would like to thank Emil Straube for his suggestion to investigate this problem and for many helpful discussions. I would also like to acknowledge Siqi Fu and thank him for some insightful conversations regarding $\left(\mathrm{CR}-P_{q}\right)$.

\section{Definitions and Notation}

\subsection{CR-Manifolds and the tangential Cauchy-Riemann operator $\bar{\partial}_{b}$.}

Definition 2.1. Let $M \subset \mathbb{C}^{N}$ be a smooth manifold of real dimension $2 n-1$. The $\boldsymbol{C R}$ structure on $M$ is given by a complex subbundle $T^{1,0}(M)$ of the complexified tangent bundle $T(M) \otimes \mathbb{C}$ that satisfies the following conditions:

(i) The complex dimension of each fiber of $T^{1,0}(M)$ is $n-1$ for all $p \in M$;

(ii) If we define $T^{0,1}(M)=\overline{T^{1,0}(M)}$, then $T^{1,0}(M) \cap T^{0,1}(M)=\{0\}$;

(iii) If $L, L^{\prime} \in T^{1,0}(M)$ are two vector fields defined near $M$, then their commutator $\left[L, L^{\prime}\right]=L L^{\prime}-L^{\prime} L$ also an element of $T^{1,0}(M)$.

A manifold $M$ endowed with a CR-structure is called a $\boldsymbol{C R}$-manifold.

In the case that $M$ is a submanifold of $\mathbb{C}^{N}$, then for each $z \in \mathbb{C}^{N}$, set $T_{z}^{1,0}(M)=T_{z}^{1,0}\left(\mathbb{C}^{N}\right) \cap$ $T_{z}(M) \otimes \mathbb{C}$ (under the natural inclusions). If the complex dimension of $T_{z}^{1,0}(M)$ is $n-1$ for all $z \in M$, we can then let $T^{1,0}(M)=\bigcup_{z \in M} T_{z}^{1,0}(M)$, and this defines the induced CR-structure on $M$. Observe that conditions (ii) and (iii) are automatically satisfied in this case.

For the remainder of this article, $M$ is a smooth, orientable CR-manifold of real dimension $2 n-1$ embedded $\mathbb{C}^{N}$ for some $N \geq n$. Let $B^{q}(M)=\bigwedge^{q}\left(T^{0,1}(M)\right)$ (the bundle of $(0, q)$ forms that consists of skew-symmetric multilinear maps of $T^{0,1}(M)^{q}$ into $\mathbb{C}$ ). We can therefore choose our Riemannian metric to be the restriction on $T(M) \otimes \mathbb{C}$ of the usual Hermitian inner product on $\mathbb{C}^{N}$. We can define a Hermitian inner product on $B^{q}(M)$ by

$$
(\varphi, \psi)=\int_{M}\langle\varphi, \psi\rangle_{x} d V
$$

where $d V$ is the volume element on $M$ and $\langle\varphi, \psi\rangle_{x}$ is the induced inner product on $B^{q}(M)$. This metric is compatible with the induced CR-structure, i.e., the vector spaces $T_{z}^{1,0}(M)$ and $T_{z}^{0,1}(M)$ are orthogonal under the inner product.

The involution condition (iii) of Definition 2.1 means that there is a restriction of the de Rham exterior derivative $d$ to $B^{q}(M)$, which we denote by $\bar{\partial}_{b}$. The inner product gives rise to an $L^{2}$-norm $\|\cdot\|_{0}$, and we also denote the closure of $\bar{\partial}_{b}$ in this norm by $\bar{\partial}_{b}$ (by an abuse of notation). In this way, $\bar{\partial}_{b}: L_{0, q}^{2}(M) \rightarrow L_{0, q+1}^{2}(M)$ is a well-defined, closed, densely defined operator, and we define $\bar{\partial}_{b}^{*}: L_{0, q+1}^{2}(M) \rightarrow L_{0, q}^{2}(M)$ to be the $L^{2}$-adjoint of $\bar{\partial}_{b}$. The Kohn Laplacian $\square_{b}: L_{0, q}^{2}(M) \rightarrow L_{0, q}^{2}(M)$ is defined as

$$
\square_{b}=\bar{\partial}_{b}^{*} \bar{\partial}_{b}+\bar{\partial}_{b} \bar{\partial}_{b}^{*},
$$


and its inverse on $(0, q)$-forms (up to $\left(\square_{b}\right)$ ) is called the complex Green operator and denoted by $G_{q}$.

The induced CR-structure has a local basis $L_{1}, \ldots, L_{n-1}$ for the $(1,0)$-vector fields in a neighborhood $U$ of each point $x \in M$. Let $\omega_{1}, \ldots, \omega_{n-1}$ be the dual basis of $(1,0)$-forms that satisfy $\left\langle\omega_{j}, L_{k}\right\rangle=\delta_{j k}$. Then $\bar{L}_{1}, \ldots, \bar{L}_{n-1}$ is a local basis for the $(0,1)$-vector fields with dual basis $\bar{\omega}_{1}, \ldots, \bar{\omega}_{n-1}$ in $U$. Also, $T(U)$ is spanned by $L_{1}, \ldots, L_{n-1}, \bar{L}_{1}, \ldots, \bar{L}_{n-1}$ and one more vector $T$ taken to be purely imaginary (so $\bar{T}=-T$ ). Let $\gamma$ be the purely imaginary global 1-form on $M$ that annihilates $T^{1,0}(M) \oplus T^{0,1}(M)$ and is normalized so that $\langle\gamma, T\rangle=-1$.

Definition 2.2. The Levi form at a point $x \in M$ is the Hermitian form given by $\left\langle d \gamma_{x}, L \wedge \bar{L}^{\prime}\right\rangle$ where $L, L^{\prime} \in T_{x}^{1,0}(U), U$ a neighborhood of $x \in M$. We call $M$ weakly pseudoconvex if there exists a form $\gamma$ such that the Levi form is positive semi-definite at all $x \in M$ and strictly pseudoconvex if there is a form $\gamma$ such that the Levi form is positive definite at all $x \in M$.

\subsection{Property $\left(\mathrm{CR}-P_{q}\right)$ and CR-plurisubharmonic functions.}

Definition 2.3. A smooth function $\varphi: \Omega \rightarrow \mathbb{C}$ is called plurisubharmonic on $(0, q)$ forms if the sum of any $q$ eigenvalues of the complex Hessian of $\varphi$ at $z \in \Omega$ is at least $C \geq 0$. The constant $C$ is the constant of plurisubharmonicity(of $\varphi$ at $z$ ).

Definition 2.4. A surface $S \subset \mathbb{R}^{k}$ satisfies property $\left(\boldsymbol{P}_{q}\right)$ if for every $C>0$, there exists a function $\varphi$ and a neighborhood $U \supset S$ so that $0 \leq \varphi \leq 1$ and $\varphi$ is plurisubharmonic on $(0, q)$-forms on $U$ with plurisubharmonicity constant $C$.

As discussed above, property $\left(P_{q}\right)$ has played a crucial role in the development of the compactness theory for the $\bar{\partial}$-Neumann operator and now we define its analog for the compactness theory of the complex Green operator on CR-manifolds of hypersurface type.

Definition 2.5. Let $M$ be a $C R$-manifold. A real-valued $\mathcal{C}_{c}^{\infty}$ function $\lambda$ defined in a neighborhood of $M$ is called strictly CR-plurisubharmonic on $(0, q)$-forms if there exist constants $A_{0}, A_{\lambda}>0$ so that for any orthonormal $Z_{j} \in T^{1,0}(M), 1 \leq j \leq q$,

$$
\sum_{j=1}^{q}\left\langle\frac{1}{2}\left(\partial_{b} \bar{\partial}_{b} \lambda-\bar{\partial}_{b} \partial_{b} \lambda\right)+A_{0} d \gamma, Z_{j} \wedge \bar{Z}_{j}\right\rangle \geq A_{\lambda}
$$

where $d \gamma$ is the invariant expression of the Levi form. $\lambda$ is called weakly CR-plurisubharmonic on $(0, q)$-forms if $A_{\lambda} \geq 0 . A_{\lambda}$ is called the $\boldsymbol{C R}$-plurisubharmonicity constant.

CR-plurisubharmonic functions were first introduced by Nicoara [Nic06] to prove closed range of $\bar{\partial}_{b}$ on $\mathrm{CR}$ manifolds of hypersurface type.

Definition 2.6. A surface $S \subset \mathbb{R}^{k}$ satisfies property $\left(\boldsymbol{C R}-\boldsymbol{P}_{q}\right)$ if for every $A>0$, there exists a function $\lambda$ and a neighborhood $U \supset S$ so that $0 \leq \lambda \leq 1$ and $\lambda$ is CR-plurisubharmonic on $(0, q)$-forms on $U$ with $C R$-plurisubharmonicity constant $A$. 
Appendix A contains results multilinear algebra that help to explain the relationship of the definitions of $\left(P_{q}\right)$ and $\left(\mathrm{CR}-P_{q}\right)$.

In this article, constants with no subscripts may depend on $n, N, M$ but not the CRplurisubharmonic functions $\lambda^{+}, \lambda^{-}$, or any quantities associated with $\lambda^{+}$or $\lambda^{-}$. Those constants will be denoted with an, $\lambda^{+}, \lambda^{-}$, or \pm in the subscript. The constant $A$ will be reserved the constant in the construction of pseudodifferential operators in Section 3 (though $A$ with subscripts will not).

\section{Computations in Local Coordinates}

3.1. Local coordinates and CR-plurisubharmonicity. The microlocal analysis that we will use relies the existence of suitable local coordinates. The first such result is Lemma 3.2 from [Nic06], recorded here as the following result.

Lemma 3.1. Let $M$ be a compact smooth, $(2 n-1)$-dimensional weakly pseudoconvex $C R$ manifold of hypersurface type embedded in a complex space $\mathbb{C}^{N}$ such that $N \geq n$ and endowed with an induced $C R$-structure. For each point $P \in M$, there exists a neighborhood $U$ so that $M \cap U$ is $C R$-equivalent to a hypersurface in $\mathbb{C}^{n}$. Additionally, on $U$ there is a local orthonormal basis $L_{1}, \ldots, L_{n}, \bar{L}_{1}, \ldots, \bar{L}_{n}$ of the $n$-dimensional complex bundle containing $T M$ :

(i) $\left.L_{j}\right|_{P}=\frac{\partial}{\partial w_{j}}$ for $1 \leq j \leq n$ where $\left(w_{1}, \ldots, w_{N}\right)$ are the coordinates of $\mathbb{C}^{N}$, and

(ii) $\left.\left[L_{j}, \bar{L}_{k}\right]\right|_{P}=c_{j k} T$ where $T=L_{n}-\bar{L}_{n}$ and $c_{j k}$ are the coefficients of the Levi form in $L_{1}, \ldots, L_{n-1}, \bar{L}_{1}, \ldots, \bar{L}_{n-1}, T$, a local basis for $T M$.

The local coordinates from Lemma 3.1 allow us to make a careful comparison of the Levi form with its $\bar{\partial}_{b}$-analog.

Proposition 3.2. Let $M$ be as in Lemma 3.1. If $\lambda$ is a smooth function near $M, L \in$ $T^{1,0}(M)$, and $\nu=L_{n}+\bar{L}_{n}$ is the "real normal" to $M$, then on $M$,

$$
\left\langle\frac{1}{2}(\partial \bar{\partial} \lambda-\bar{\partial} \partial \lambda), L \wedge \bar{L}\right\rangle-\left\langle\frac{1}{2}\left(\partial_{b} \bar{\partial}_{b} \lambda-\bar{\partial}_{b} \partial_{b} \lambda\right), L \wedge \bar{L}\right\rangle=\frac{1}{2} \nu\{\lambda\}\langle d \gamma, L \wedge \bar{L}\rangle
$$

Proof. Using Lemma 3.1, there exists a basis of $\mathbb{C} T\left(\mathbb{C}^{N}\right)$ given by $L_{1}, \ldots, L_{N}, \bar{L}_{1}, \ldots, \bar{L}_{N}$ so that $L_{1}, \ldots, L_{n-1}$ and $\bar{L}_{1}, \ldots, \bar{L}_{n-1}$ are a basis of $T^{1,0}(M)$ and $T^{0,1}(M)$, respectively, $T=L_{n}-\bar{L}_{n} \in T M$ is a purely imaginary tangent vector, and $\nu=L_{n}+\bar{L}_{n}$ is the "real normal" tangent vector to $M$. Let $\omega_{1}, \ldots, \omega_{N}, \bar{\omega}_{1}, \ldots, \bar{\omega}_{N}$ be the dual cotangent vectors to $L_{1}, \ldots, L_{N}, \bar{L}_{1}, \ldots, \bar{L}_{N}$, respectively. Assume that the coordinates are centered around $P \in M$ in sense of Lemma 3.1.

Recall that $\partial \bar{\partial}=-\bar{\partial} \partial$, so $\partial \bar{\partial}=\frac{1}{2}(\partial \bar{\partial}-\bar{\partial} \partial)$. We now compute

$$
\partial \bar{\partial} \lambda=\partial\left(\sum_{k=1}^{N} \bar{L}_{k} \lambda \bar{\omega}_{k}\right)=\sum_{j, k=1}^{N} L_{j} \bar{L}_{k} \lambda \omega_{j} \wedge \bar{\omega}_{k}+\sum_{\ell=1}^{N} \bar{L}_{\ell} \lambda \partial \bar{\omega}_{\ell}
$$


Also,

$$
\bar{\partial} \partial \lambda=\bar{\partial}\left(\sum_{j=1}^{N} L_{j} \lambda \omega_{j}\right)=-\sum_{j, k=1}^{N} \bar{L}_{k} L_{j} \lambda \omega_{j} \wedge \bar{\omega}_{k}+\sum_{\ell=1}^{N} L_{\ell} \lambda \bar{\partial} \omega_{\ell}
$$

Let $L=\sum_{j=1}^{n-1} \xi_{j} L_{j}$ be a complex tangent vector on $M$. Then

$$
\begin{aligned}
\left.\left\langle\bar{\partial} \omega_{\ell}, L_{j} \wedge \bar{L}_{k}\right\rangle\right|_{P} & =\left.L_{j}\left\{\left\langle\omega_{\ell}, \bar{L}_{k}\right\rangle\right\}\right|_{P}-\left.\bar{L}_{j}\left\{\left\langle\omega_{\ell}, L_{k}\right\rangle\right\}\right|_{P}-\left.\left\langle\omega_{\ell},\left[L_{j}, \bar{L}_{k}\right]\right\rangle\right|_{P} \\
& =-\left\langle\left.\left(\omega_{\ell}, c_{j k} T\right\rangle\right|_{P}=-\delta_{\ell n} c_{j k}(P) .\right.
\end{aligned}
$$

Similarly, since $T=L_{n}-\bar{L}_{n}$,

$$
\begin{aligned}
\left.\left\langle\partial \bar{\omega}_{\ell}, L_{j} \wedge \bar{L}_{k}\right\rangle\right|_{P} & =\left.L_{j}\left\{\left\langle\bar{\omega}_{\ell}, \bar{L}_{k}\right\rangle\right\}\right|_{P}-\left.\bar{L}_{j}\left\{\left\langle\bar{\omega}_{\ell}, L_{k}\right\rangle\right\}\right|_{P}-\left.\left\langle\bar{\omega}_{\ell},\left[L_{j}, \bar{L}_{k}\right]\right\rangle\right|_{P} \\
& =-\left.\left\langle\bar{\omega}_{\ell}, c_{j k} T\right\rangle\right|_{P}=\delta_{\ell n} c_{j k}(P) .
\end{aligned}
$$

Consequently, for $1 \leq j, k \leq n-1$,

$$
\left.\left\langle\sum_{\ell=1}^{N}\left(\bar{L}_{\ell} \lambda \partial \bar{\omega}_{\ell}-L_{\ell} \lambda \partial \bar{\omega}_{\ell}\right), L_{j} \wedge \bar{L}_{k}\right\rangle\right|_{P}=\left(\bar{L}_{n}\{\lambda\}+L_{n}\{\lambda\}\right) c_{j k}(P)=\left.c_{j k}(P) \nu(\lambda)\right|_{P}
$$

If $K=\sum_{j=1}^{N} \xi_{j} L_{j}+\sum_{k=1}^{N} \zeta_{k} \bar{L}_{k}$, then

$$
\left\langle\omega_{j} \wedge \bar{\omega}_{k}, K \wedge \bar{K}\right\rangle=\omega_{j}(K) \bar{\omega}_{k}(\bar{K})-\omega_{j}(\bar{K}) \bar{\omega}_{k}(K)=\xi_{j} \bar{\xi}_{k}-\bar{\zeta}_{j} \zeta_{k} .
$$

Putting the equations together, for $L=\sum_{j=1}^{n-1} \xi_{j} L_{j}$, we have that

$$
\left.\langle\partial \bar{\partial} \lambda, L \wedge \bar{L}\rangle\right|_{P}=\left.\left\langle\frac{1}{2}(\partial \bar{\partial} \lambda-\bar{\partial} \partial \lambda), L \wedge \bar{L}\right\rangle\right|_{P}=\left.\frac{1}{2} \sum_{j, k=1}^{n-1}\left[L_{j} \bar{L}_{k} \lambda+\bar{L}_{k} L_{j} \lambda+\nu(\lambda) c_{j k}\right] \xi_{j} \bar{\xi}_{k}\right|_{P} .
$$

To understand $L_{j} \bar{L}_{k} \lambda+\bar{L}_{k} L_{j} \lambda$, we expand the vector fields in the ambient $\mathbb{C}^{N}$ coordinates. In coordinates,

$$
L_{j}=\sum_{\ell=1}^{N} a_{\ell}^{j} \frac{\partial}{\partial w_{\ell}}
$$

This means

$$
L_{j} \bar{L}_{k}-\sum_{\ell, \ell^{\prime}=1}^{N} a_{\ell}^{j} \bar{a}_{\ell^{\prime}}^{k} \frac{\partial^{2}}{\partial w_{\ell} \partial \bar{w}_{\ell^{\prime}}}=\sum_{\ell, \ell^{\prime}=1}^{N} a_{\ell}^{j} \frac{\partial \bar{a}_{\ell^{\prime}}^{k}}{\partial w_{\ell}} \frac{\partial}{\partial \bar{w}_{\ell^{\prime}}} \in T^{0,1}\left(\mathbb{C}^{N}\right)
$$

and

$$
\bar{L}_{k} L_{j}-\sum_{\ell, \ell^{\prime}=1}^{N} a_{\ell}^{j} \bar{a}_{\ell^{\prime}}^{k} \frac{\partial^{2}}{\partial w_{\ell} \partial \bar{w}_{\ell^{\prime}}}=\sum_{\ell, \ell^{\prime}=1}^{N} \bar{a}_{\ell^{\prime}}^{k} \frac{\partial a_{\ell}^{j}}{\partial \bar{w}_{\ell^{\prime}}} \frac{\partial}{\partial w_{\ell}} \in T^{1,0}\left(\mathbb{C}^{N}\right)
$$

Since $\left.\left[L_{j}, \bar{L}_{k}\right]\right|_{P}=L_{j} \bar{L}_{k}-\left.\bar{L}_{k} L_{j}\right|_{P}=\left.c_{j k} T\right|_{P}=\left.c_{j k}\left(L_{n}-\bar{L}_{n}\right)\right|_{P}$, it follows that

$$
\left.\sum_{\ell, \ell^{\prime}=1}^{N} \bar{a}_{\ell^{\prime}}^{k} \frac{\partial a_{\ell}^{j}}{\partial \bar{w}_{\ell^{\prime}}} \frac{\partial}{\partial w_{\ell}}\right|_{P}=-\left.c_{j k} L_{n}\right|_{P}
$$


and

$$
\left.\sum_{\ell, \ell^{\prime}=1}^{N} a_{\ell}^{j} \frac{\partial \bar{a}_{\ell^{\prime}}^{k}}{\partial w_{\ell}} \frac{\partial}{\partial \bar{w}_{\ell^{\prime}}}\right|_{P}=-\left.c_{j k} \bar{L}_{n}\right|_{P} .
$$

Thus, since $\left.L_{j}\right|_{P}=\frac{\partial}{\partial w_{j}}$ by Lemma 3.1,

$$
\left.\left(L_{j} \bar{L}_{k} \lambda+\bar{L}_{k} L_{j} \lambda\right)\right|_{P}=\frac{\partial^{2} \lambda(P)}{\partial w_{j} \partial \bar{w}_{k}}-\left.\nu(\lambda)\right|_{P} c_{j k}(P) .
$$

Finally,

$$
\begin{aligned}
\left.\left\langle\frac{1}{2}(\partial \bar{\partial} \lambda-\bar{\partial} \partial \lambda), L \wedge \bar{L}\right\rangle\right|_{P} & =\left.\frac{1}{2} \sum_{j, k=1}^{n-1}\left[\left(\frac{\partial^{2} \lambda}{\partial w_{j} \partial \bar{w}_{k}}+c_{j k}(\nu(\lambda)-\nu(\lambda))\right) \xi_{j} \bar{\xi}_{k}\right]\right|_{P} \\
& =\left.\frac{1}{2} \sum_{j, k=1}^{n-1} \frac{\partial^{2} \lambda}{\partial w_{j} \partial \bar{w}_{k}} \xi_{j} \bar{\xi}_{k}\right|_{P}
\end{aligned}
$$

The calculation of $\left.\left\langle\frac{1}{2}\left(\partial_{b} \bar{\partial}_{b} \lambda-\bar{\partial}_{b} \partial_{b} \lambda\right), L \wedge \bar{L}\right\rangle\right|_{P}$ is performed identically except that the sums in (11) and (2) only go to $n-1$ and not to $N$. The result is that

$$
\left.\left\langle\frac{1}{2}\left(\partial_{b} \bar{\partial}_{b} \lambda-\bar{\partial}_{b} \partial_{b} \lambda\right), L \wedge \bar{L}\right\rangle\right|_{P}=\left.\frac{1}{2} \sum_{j, k=1}^{n-1}\left[\left(\frac{\partial^{2} \lambda}{\partial w_{j} \partial \bar{w}_{k}}-c_{j k} \nu(\lambda)\right) \xi_{j} \bar{\xi}_{k}\right]\right|_{P} .
$$

Consequently,

$$
\begin{aligned}
\left.\left\langle\frac{1}{2}(\partial \bar{\partial} \lambda-\bar{\partial} \partial \lambda), L \wedge \bar{L}\right\rangle\right|_{P}-\left.\left\langle\frac{1}{2}\left(\partial_{b} \bar{\partial}_{b} \lambda-\bar{\partial}_{b} \partial_{b} \lambda\right), L \wedge \bar{L}\right\rangle\right|_{P} & =\left.\frac{1}{2} \sum_{j, k=1}^{n-1} c_{j k} \nu(\lambda) \xi_{j} \bar{\xi}_{k}\right|_{P} \\
& =\left.\frac{1}{2} \nu(\lambda)\langle d \gamma, L \wedge \bar{L}\rangle\right|_{P}
\end{aligned}
$$

However, $T$ and $d \gamma$ are globally defined quantities and $P$ was arbitrary, so on $M$,

$$
\left\langle\frac{1}{2}(\partial \bar{\partial} \lambda-\bar{\partial} \partial \lambda), L \wedge \bar{L}\right\rangle-\left\langle\frac{1}{2}\left(\partial_{b} \bar{\partial}_{b} \lambda-\bar{\partial}_{b} \partial_{b} \lambda\right), L \wedge \bar{L}\right\rangle=\frac{1}{2} \nu\{\lambda\}\langle d \gamma, L \wedge \bar{L}\rangle
$$

We can already see from Proposition 3.2 the importance of CR-plurisubharmonic functions. On a compact (smooth) manifold, $\nu\{\lambda\}$ will be a bounded quantity, and multiples of Leviform are controlled by CR-plurisubharmonicity.

If $\lambda$ is smooth function defined near $P \in M$, let $\lambda_{j k}$ satisfy

$$
\partial \bar{\partial} \lambda=\sum_{j, k=1}^{N} \lambda_{j k} \omega_{j} \wedge \bar{\omega}_{k}
$$

Also, let $\mathcal{I}_{q}=\left\{J=\left(j_{1}, \ldots, j_{q}\right) \in \mathbb{N}^{q}: 1 \leq j_{1}<\cdots<j_{q} \leq n\right\}$ and $\mathcal{I}_{q}^{\prime}=\left\{J \in \mathcal{I}_{q}: j_{q}<n\right\}$. 
As a of Proposition 3.2 and Lemma A.1, we learn that functions that are plurisubharmonic on $(0, q)$-forms near $M$ are CR-plurisubharmonic on $(0, q)$-forms.

Corollary 3.3. Let $M$ be as in Lemma 3.1. If $\lambda$ is a smooth, real-valued function that is plurisubharmonic on $(0, q)$-forms near $M$ and has $C R$-plurisubharmonicity constant $A_{\lambda}$, then $\lambda$ is CR-plurisubharmonic on $(0, q)$-forms with CR-plurisubharmonicity constant $A_{\lambda}$.

3.2. Pseudodifferential Operators. From Lemma 3.1, there exists a finite cover $\left\{U_{\nu}\right\}_{\nu}$ so each $U_{\nu}$ has a special boundary system and can be parameterized by a hypersurface in $\mathbb{C}^{n}\left(U_{\nu}\right.$ may be shrunk as necessary). To set up the microlocal analysis, we need to define the appropriate pseudodifferential operators on each $U_{\nu}$. Let $\xi=\left(\xi_{1}, \ldots, \xi_{2 n-2}, \xi_{2 n-1}\right)=$ $\left(\xi^{\prime}, \xi_{2 n-1}\right)$ be the coordinates in Fourier space so that $\xi^{\prime}$ is dual to the part of $T(M)$ in the maximal complex subspace (i.e., $\left.T^{1,0}(M) \oplus T^{0,1}(M)\right)$ and $\xi_{2 n-1}$ is dual to the totally real part of $T(M)$, i.e., the "bad" direction $T$. Define

$$
\begin{aligned}
& \mathcal{C}^{+}=\left\{\xi: \xi_{2 n-1} \geq \frac{1}{2}\left|\xi^{\prime}\right| \text { and }|\xi| \geq 1\right\} ; \\
& \mathcal{C}^{-}=\left\{\xi:-\xi \in \mathcal{C}^{+}\right\} \\
& \mathcal{C}^{0}=\left\{\xi:-\frac{3}{4}\left|\xi^{\prime}\right| \leq \xi_{2 n-1} \leq \frac{3}{4}\left|\xi^{\prime}\right|\right\} \cup\{\xi:|\xi| \leq 1\} .
\end{aligned}
$$

Note that $\mathcal{C}^{+}$and $\mathcal{C}^{-}$are disjoint, but both intersect $\mathcal{C}^{0}$ nontrivially. Next, we define functions on $\left\{|\xi|:|\xi|^{2}=1\right\}$. Let

$$
\begin{aligned}
& \psi^{+}(\xi)=1 \text { when } \xi_{2 n-1} \geq \frac{3}{4}\left|\xi^{\prime}\right| \text { and } \operatorname{supp} \psi^{+} \subset\left\{\xi: \xi_{2 n-1} \geq \frac{1}{2}\left|\xi^{\prime}\right|\right\} \\
& \psi^{-}(\xi)=\psi^{+}(-\xi) \\
& \psi^{0}(\xi) \text { satisfies } \psi^{0}(\xi)^{2}=1-\psi^{+}(\xi)^{2}-\psi^{-}(\xi)^{2} .
\end{aligned}
$$

Extend $\psi^{+}, \psi^{-}$, and $\psi^{0}$ homogeneously outside of the unit ball, i.e., if $|\xi| \geq 1$, then

$$
\psi^{+}(\xi)=\psi^{+}(\xi /|\xi|), \psi^{-}(\xi)=\psi^{-}(\xi /|\xi|), \text { and } \psi^{0}(\xi)=\psi^{0}(\xi /|\xi|) .
$$

Also, extend $\psi^{+}, \psi^{-}$, and $\psi^{0}$ smoothly inside the unit ball so that $\left(\psi^{+}\right)^{2}+\left(\psi^{-}\right)^{2}+\left(\psi^{0}\right)^{2}=1$. Finally, for $A$ to be chosen later, define

$$
\psi_{A}^{+}(\xi)=\psi(\xi / A), \psi_{A}^{-}(\xi)=\psi^{-}(\xi / A) \text {, and } \psi_{A}^{0}(\xi)=\psi^{0}(\xi / A) .
$$

Next, let $\Psi_{A}^{+}, \Psi_{A}^{-}$, and $\Psi^{0}$ be the pseudodifferential operators of order zero with symbols $\psi_{A}^{+}$, $\psi_{A}^{-}$, and $\psi_{A}^{0}$, respectively. The equality $\left(\psi_{A}^{+}\right)^{2}+\left(\psi_{A}^{-}\right)^{2}+\left(\psi_{A}^{0}\right)^{2}=1$ implies that

$$
\left(\Psi_{A}^{+}\right)^{*} \Psi_{A}^{+}+\left(\Psi_{A}^{0}\right)^{*} \Psi_{A}^{0}+\left(\Psi_{A}^{-}\right)^{*} \Psi_{A}^{-}=I d .
$$

We will also have use for pseudodifferential operators that "dominate" a given pseudodifferential operator. Let $\psi$ be cut-off function and $\tilde{\psi}$ be another cut-off function so that $\left.\tilde{\psi}\right|_{\text {supp } \psi} \equiv 1$. If $\Psi$ and $\tilde{\Psi}$ are pseudodifferential operators with symbols $\psi$ and $\tilde{\psi}$, respectively, then we say that $\tilde{\Psi}$ dominates $\Psi$.

For each $U_{\nu}$, we have a local CR-equivalence to a hypersurface in $\mathbb{C}^{n}$, and we can define $\Psi_{A}^{+}, \Psi_{A}^{-}$, and $\Psi_{A}^{0}$ to act on functions or forms supported in $U_{\nu}$, so let $\Psi_{\nu, A}^{+}, \Psi_{\nu, A}^{-}$, and $\Psi_{\nu, A}^{0}$ 
be the pseudodifferential operators of order zero defined on $U_{\nu}$ and $\mathcal{C}_{\nu}^{+}$, and $\mathcal{C}_{\nu}^{-}$, and $\mathcal{C}_{\nu}^{0}$ be the regions of $\xi$-space dual to $U_{\nu}$ on which the symbol of each of those pseudodifferential operators is supported. Then it follows that:

$$
\left(\Psi_{\nu, A}^{+}\right)^{*} \Psi_{\nu, A}^{+}+\left(\Psi_{\nu, A}^{0}\right)^{*} \Psi_{\nu, A}^{0}+\left(\Psi_{\nu, A}^{-}\right)^{*} \Psi_{\nu, A}^{-}=I d .
$$

Let $\tilde{\Psi}_{\mu, A}^{+}$and $\tilde{\Psi}_{\mu, A}^{-}$be pseudodifferential operators that dominate $\Psi_{\mu, A}^{+}$and $\Psi_{\mu, A}^{-}$, respectively (where $\Psi_{\mu, A}^{+}$and $\Psi_{\mu, A}^{-}$are defined on some $U_{\mu}$ ). If $\tilde{\mathcal{C}}_{\mu}^{+}$and $\tilde{\mathcal{C}}_{\mu}^{-}$are the supports of $\tilde{\Psi}_{\mu, A}^{+}$and $\tilde{\Psi}_{\mu, A}^{-}$, respectively, then we can choose $\left\{U_{\mu}\right\}, \tilde{\psi}_{\mu, A}^{+}$, and $\tilde{\psi}_{\mu, A}^{-}$so that the following result holds.

Lemma 3.4. Let $M$ be a compact, orientable, embedded CR-manifold. There is a finite open covering $\left\{U_{\mu}\right\}_{\mu}$ of $M$ so that if $U_{\mu}, U_{\nu} \in\left\{U_{\mu}\right\}$ have nonempty intersection, then there exists a diffeomorphism $\vartheta$ between $U_{\nu}$ and $U_{\mu}$ with Jacobian $\mathcal{J}_{\vartheta}$ so that:

(1) ${ }^{t} \mathcal{J}_{\vartheta}\left(\tilde{\mathcal{C}}_{\mu}^{+}\right) \cap \mathcal{C}_{\nu}^{-}=\emptyset$ and $\mathcal{C}_{\nu}^{+} \cap{ }^{t} \mathcal{J}_{\vartheta}\left(\tilde{\mathcal{C}}_{\mu}^{-}\right)=\emptyset$ where ${ }^{t} \mathcal{J}_{\vartheta}$ is the inverse of the transpose of $\mathcal{J}_{\vartheta}$

(2) Let ${ }^{\vartheta} \Psi_{\mu, A}^{+},{ }^{\vartheta} \Psi_{\mu, A}^{-}$, and ${ }^{\vartheta} \Psi_{\mu, A}^{0}$ be the transfers of $\Psi_{\mu, A}^{+}, \Psi_{\mu, A}^{-}$, and $\Psi_{\mu, A}^{0}$, respectively via $\vartheta$. Then on $\left\{\xi: \xi_{2 n-1} \geq \frac{4}{5}\left|\xi^{\prime}\right|\right.$ and $\left.|\xi| \geq(1+\epsilon) A\right\}$, then principal symbol of ${ }^{\vartheta} \Psi_{\mu, A}^{+}$ is identically 1 , on $\left\{\xi: \xi_{2 n-1} \leq-\frac{4}{5}\left|\xi^{\prime}\right|\right.$ and $\left.|\xi| \geq(1+\epsilon) A\right\}$, then principal symbol of ${ }^{\vartheta} \Psi_{\mu, A}^{-}$is identically 1 , and on $\left\{\xi:-\frac{1}{3} \xi_{2 n-1} \geq \frac{1}{3}\left|\xi^{\prime}\right|\right.$ and $\left.|\xi| \geq(1+\epsilon) A\right\}$, then principal symbol of ${ }^{\vartheta} \Psi_{\mu, A}^{0}$ is identically 1 , where $\epsilon>0$ and can be very small;

(3) Let ${ }^{\vartheta} \tilde{\Psi}_{\mu, A}^{+}$, $\vartheta \tilde{\Psi}_{\mu, A}^{-}$be the transfers via $\vartheta$ of $\tilde{\Psi}_{\mu, A}^{+}$and $\tilde{\Psi}_{\mu, A}^{-}$, respectively. Then the principal symbol of $\vartheta \tilde{\Psi}_{\mu, A}^{+}$is identically 1 on $\mathcal{C}_{\nu}^{+}$and the principal symbol of ${ }^{\vartheta} \tilde{\Psi}_{\mu, A}^{-}$is identically 1 on $\mathcal{C}_{\nu}^{-}$

(4) $\tilde{\mathcal{C}}_{\mu}^{+} \cap \tilde{\mathcal{C}}_{\mu}^{-}=\emptyset$.

We will suppress the left superscript $\vartheta$ as it should be clear from the context which pseudodifferential operator must be transferred. The proof of this lemma is contained in Lemma 4.3 and its subsequent discussion in [Nic06] .

3.3. Norms. We have a volume form $d V$ on $M$, and we define the following inner products and norms on functions (with their natural generalizations to forms). Let $\lambda^{+}$and $\lambda^{-}$be functions defined on $M$.

$$
\begin{aligned}
(\phi, \varphi)_{0} & =\int_{M} \phi \bar{\varphi} d V, \text { and }\|\varphi\|_{0}^{2}=(\varphi, \varphi)_{0} \\
(\phi, \varphi)_{\lambda^{+}} & =\int_{M} \phi \bar{\varphi} e^{-\lambda^{+}} d V, \text { and }\|\varphi\|_{\lambda^{+}}^{2}=(\varphi, \varphi)_{\lambda^{+}} \\
(\phi, \varphi)_{\lambda^{-}} & =\int_{M} \phi \bar{\varphi} e^{\lambda^{-}} d V, \text { and }\|\varphi\|_{\lambda^{-}}^{2}=(\varphi, \varphi)_{\lambda^{-}} .
\end{aligned}
$$

If $\varphi=\sum_{j \in \mathcal{I}_{q}^{\prime}} \varphi_{j} \bar{\omega}_{J}$, then we use the common shorthand $\|\varphi\|=\sum_{j \in \mathcal{I}_{q}^{\prime}}\left\|\varphi_{J}\right\|$ where $\|\cdot\|$ represents a generic norm norm applied to $\varphi$. 
We also need a norm that is well-suited for the microlocal arguments. Let $\left\{\zeta_{\nu}\right\}$ be a partition of unity subordinate to the covering $\left\{U_{\nu}\right\}$ satisfying $\sum_{\nu} \zeta_{\nu}^{2}=1$. Also, for each $\nu$, let $\tilde{\zeta}_{\nu}$ be a cutoff function that dominates $\zeta_{\nu}$ so that $\operatorname{supp} \tilde{\zeta}_{\nu} \subset U_{\nu}$. Then we define the global inner product and norm as follows:

$$
\begin{aligned}
\langle\phi, \varphi\rangle_{\lambda^{+}, \lambda^{-}}=\langle\phi, \varphi\rangle_{ \pm}=\sum_{\nu} & \left(\left(\tilde{\zeta}_{\nu} \Psi_{\nu, A}^{+} \zeta_{\nu} \phi^{\nu}, \tilde{\zeta}_{\nu} \Psi_{\nu, A}^{+} \zeta_{\nu} \varphi^{\nu}\right)_{\lambda^{+}}\right. \\
& \left.+\left(\tilde{\zeta}_{\nu} \Psi_{\nu, A}^{0} \zeta_{\nu} \phi^{\nu}, \tilde{\zeta}_{\nu} \Psi_{\nu, A}^{0} \zeta_{\nu} \varphi^{\nu}\right)_{0}+\left(\tilde{\zeta}_{\nu} \Psi_{\nu, A}^{-} \zeta_{\nu} \phi^{\nu}, \tilde{\zeta}_{\nu} \Psi_{\nu, A}^{-} \zeta_{\nu} \varphi^{\nu}\right)_{\lambda^{-}}\right)
\end{aligned}
$$

and

$$
\|\varphi \varphi\|_{\lambda^{+}, \lambda^{-}}^{2}=\|\| \varphi \|_{ \pm}^{2}=\sum_{\nu}\left(\left\|\tilde{\zeta}_{\nu} \Psi_{\nu, A}^{+} \zeta_{\nu} \varphi^{\nu}\right\|_{\lambda^{+}}^{2}+\left\|\tilde{\zeta}_{\nu} \Psi_{\nu, A}^{0} \zeta_{\nu} \varphi^{\nu}\right\|_{0}^{2}+\left\|\tilde{\zeta}_{\nu} \Psi_{\nu, A}^{-} \zeta_{\nu} \varphi^{\nu}\right\|_{\lambda^{-}}^{2}\right)
$$

where $\varphi^{\nu}$ is the form $\varphi$ expressed in the local coordinates on $U_{\nu}$. The superscript $\nu$ will often be omitted.

For a form $\varphi$ supported on $M$, the Sobolev norm of order $s$ is given by the following:

$$
\|\varphi\|_{s}^{2}=\sum_{\nu}\left\|\tilde{\zeta}_{\nu} \Lambda^{s} \zeta_{\nu} \varphi^{\nu}\right\|_{0}^{2}
$$

where $\Lambda$ is defined to be the pseudodifferential operator with symbol $\left(1+|\xi|^{2}\right)^{1 / 2}$.

It will be essential for us to pass from a the unweighted $L^{2}$-norm on $M$ and the microlocal norm defined above. The following lemma says that we can do this without any loss of information.

Lemma 3.5. Let $\lambda^{+}, \lambda^{-}$be smooth functions on $M$ with $0 \leq \lambda^{+}, \lambda^{-} \leq 1$. Then there exist constants $C_{1}, C_{2}>0$ so that

$$
C_{1}\|\varphi\|_{0}^{2} \leq\|\varphi\|_{ \pm}^{2} \leq C_{2}\|\varphi\|_{0}^{2}
$$

Proof. It is enough to check this when $\varphi$ is a function. Since $0 \leq \lambda^{+}, \lambda^{-} \leq 1$,

$$
\|\varphi\|_{ \pm}^{2} \leq e \sum_{\nu}\left(\left\|\tilde{\zeta}_{\nu} \Psi_{\nu, A}^{+} \zeta_{\nu} \varphi^{\nu}\right\|_{0}^{2}+\left\|\tilde{\zeta}_{\nu} \Psi_{\nu, A}^{0} \zeta_{\nu} \varphi^{\nu}\right\|_{0}^{2}+\left\|\tilde{\zeta}_{\nu} \Psi_{\nu, A}^{-} \zeta_{\nu} \varphi^{\nu}\right\|_{0}^{2}\right)
$$

We can express $\tilde{\zeta}_{\nu} \Psi_{\nu, A}^{+} \zeta_{\nu} \varphi^{\nu}=\Psi_{\nu, A}^{+} \zeta_{\nu} \varphi^{\nu}-\left(1-\tilde{\zeta}_{\nu}\right) \Psi_{\nu, A}^{+} \zeta_{\nu} \varphi^{\nu} . \quad\left(1-\tilde{\zeta}_{\nu}\right) \Psi_{\nu, A}^{+} \zeta_{\nu}$ is infinitely smoothing, but using this bound would lead to a constant depending on $A$. We wish to avoid constants depending on $A$. Observe that

$$
\begin{aligned}
\left(1-\tilde{\zeta}_{\nu}(x)\right) \Psi_{\nu, A}^{+} \zeta_{\nu}(x) \varphi^{\nu}(x) & =\frac{1}{(2 \pi)^{2 n-1}}\left(1-\tilde{\zeta}_{\nu}(x)\right) \int_{\mathbb{R}^{2 n-1}} e^{i x \cdot \xi} \psi_{\nu, A}^{+}(\xi) \widehat{\zeta_{\nu} \varphi^{\nu}}(\xi) d \xi \\
= & \frac{1}{(2 \pi)^{2 n-1}} \int_{\mathbb{R}^{2 n-1}} \varphi^{\nu}(y) \int_{\mathbb{R}^{2 n-1}}\left(1-\tilde{\zeta}_{\nu}(x)\right) \zeta_{\nu}(y) e^{i(x-y) \cdot \xi} \psi_{\nu, A}^{+}(\xi) d \xi d y
\end{aligned}
$$

Define $K(x, y)=\frac{1}{(2 \pi)^{2 n-1}} \int_{\mathbb{R}^{2 n-1}}\left(1-\tilde{\zeta}_{\nu}(x)\right) \zeta_{\nu}(y) e^{i(x-y) \cdot \xi} \psi_{\nu, A}^{+}(\xi) d \xi$. By integration by parts, for any multiindex $\alpha$,

$$
K(x, y)=\left(1-\tilde{\zeta}_{\nu}(x)\right) \zeta_{\nu}(y) \frac{(-i)^{\alpha}}{\left(2 \pi(x-y)^{\alpha}\right)^{2 n-1}} \int_{\mathbb{R}^{2 n-1}} e^{i(x-y) \cdot \xi} D^{\alpha} \psi_{\nu, A}^{+}(\xi) d \xi
$$


Recall that $\psi_{\nu, A}^{+}(\xi)=\psi^{+}(\xi / A)$, so requiring that $A \geq 1$ means that $\left|D^{\alpha} \psi_{\nu, A}^{+}(\xi)\right| \leq C_{\alpha}$ where $C_{\alpha}$ does not depend on $A$. However, $\operatorname{supp}\left(1-\tilde{\zeta}_{\nu}\right) \cap \operatorname{supp} \zeta_{\nu}=\emptyset$, so for any $N$, there exists $C_{N}$ so that

$$
|K(x, y)| \leq\left|1-\tilde{\zeta}_{\nu}(x)\right|\left|\zeta_{\nu}(y)\right| \frac{C_{N}}{(1+|x-y|)^{N}},
$$

where $C_{N}$ does not depend on $A$. Consequently,

$$
\left\|\left(1-\tilde{\zeta}_{\nu}\right) \Psi_{\nu, A}^{+} \zeta_{\nu} \varphi^{\nu}(x)\right\|_{0}^{2} \leq \tilde{C}\left\|\zeta_{\nu} \varphi^{\nu}\right\|_{0}^{2} .
$$

The range of $\Psi_{\nu, A}^{+} \zeta_{\nu}$ is not $L^{2}\left(U_{\nu}\right)$ but $L^{2}\left(\mathbb{R}^{2 n-1}\right)$, but this problem is mitigated by the fact that $\Psi_{\nu, A}^{+} \zeta_{\nu}$ is a smoothing operator outside of $\operatorname{Dom}\left(\zeta_{\nu}\right)$. Also, $\Psi_{A}^{+} \zeta_{\nu}$ is a contraction on $L^{2}\left(\mathbb{R}^{2 n-1}\right)$, so

$$
\left\|\tilde{\zeta}_{\nu} \Psi_{\nu, A}^{+} \zeta_{\nu} \varphi^{\nu}\right\|_{0}^{2} \leq 2\left\|\Psi_{\nu, A}^{+} \zeta_{\nu} \varphi^{\nu}\right\|_{0}^{2}+2\left\|\left(1-\tilde{\zeta}_{\nu}\right) \Psi_{\nu, A}^{+} \zeta_{\nu} \varphi^{\nu}\right\|_{0}^{2} \leq C_{+}\left\|\zeta_{\nu} \varphi^{\nu}\right\|_{0}^{2}
$$

for some $C$ independent of $A$. By (possibly) increasing $C$, a similar bound will also hold for for $\Psi_{\nu, A}^{0}$ and $\Psi_{\nu, A}^{-}$. The upper bound of the lemma therefore follows (since the sum over $\nu$ is finite and $\left.0 \leq \zeta_{\nu} \leq 1\right)$.

We now show the lower bound. Note that $\sum_{\nu} \zeta_{\nu}^{2}=1=\sum_{\nu} \tilde{\zeta}_{\nu} \zeta_{\nu}^{2}$. Consequently,

$$
\begin{aligned}
& \|\varphi\|_{0}^{2}=\left(\sum_{\nu} \zeta_{\nu}^{2} \varphi, \varphi\right)_{0}=\sum_{\nu}\left\|\zeta_{\nu} \varphi^{\nu}\right\|_{0}^{2} \\
& =\sum_{\nu}\left(\left(\left(\Psi_{\nu, A}^{+}\right)^{*} \Psi_{\nu, A}^{+}+\left(\Psi_{\nu, A}^{0}\right)^{*} \Psi_{\nu, A}^{0}+\left(\Psi_{\nu, A}^{-}\right)^{*} \Psi_{\nu, A}^{-}\right) \varphi^{\nu}, \varphi^{\nu}\right)_{0} \\
& =\sum_{\nu}\left(\left\|\left(\tilde{\zeta}_{\nu}+\left(1-\tilde{\zeta}_{\nu}\right)\right) \Psi_{\nu, A}^{+} \zeta_{\nu} \varphi^{\nu}\right\|_{0}^{2}+\left\|\left(\tilde{\zeta}_{\nu}+\left(1-\tilde{\zeta}_{\nu}\right)\right) \Psi_{\nu, A}^{0} \zeta_{\nu} \varphi^{\nu}\right\|_{0}^{2}+\left\|\left(\tilde{\zeta}_{\nu}+\left(1-\tilde{\zeta}_{\nu}\right)\right) \Psi_{\nu, A}^{-} \zeta_{\nu} \varphi^{\nu}\right\|_{0}^{2}\right)
\end{aligned}
$$

However, $\left\|\left(\tilde{\zeta}_{\nu}+\left(1-\tilde{\zeta}_{\nu}\right)\right) \Psi_{\nu, A}^{+} \zeta_{\nu} \varphi^{\nu}\right\|_{0}^{2} \leq 2\left(\left\|\tilde{\zeta}_{\nu} \Psi_{\nu, A}^{+} \zeta_{\nu} \varphi^{\nu}\right\|_{0}^{2}+\left\|\left(1-\tilde{\zeta}_{\nu}\right) \Psi_{\nu, A}^{+} \zeta_{\nu} \varphi^{\nu}\right\|_{0}^{2}\right)$, and $\Psi_{\nu, A}^{+} \zeta_{\nu} \varphi^{\nu}$ is pseudolocal (indeed, $\left.\left(1-\tilde{\zeta}_{\nu}\right)\right) \Psi_{\nu, A}^{+} \zeta_{\nu} \varphi^{\nu}$ is infinitely smoothing), so $\left\|\tilde{\zeta}_{\nu} \Psi_{\nu, A}^{+} \zeta_{\nu} \varphi^{\nu}\right\|_{0}^{2}$ controls $\left\|\left(1-\tilde{\zeta}_{\nu}\right) \Psi_{\nu, A}^{+} \zeta_{\nu} \varphi^{\nu}\right\|_{0}^{2}$ and similarly for $\Psi_{\nu, A}^{-}$and $\Psi_{\nu, A}^{0}$. As a result,

$$
\begin{aligned}
\|\varphi\|_{0}^{2} & \leq C \sum_{\nu}\left(\left\|\tilde{\zeta}_{\nu} \Psi_{\nu, A}^{+} \zeta_{\nu} \varphi^{\nu}\right\|_{0}^{2}+\left\|\tilde{\zeta}_{\nu} \Psi_{\nu, A}^{0} \zeta_{\nu} \varphi^{\nu}\right\|_{0}^{2}+\left\|\tilde{\zeta}_{\nu} \Psi_{\nu, A}^{-} \zeta_{\nu} \varphi^{\nu}\right\|_{0}^{2}\right) \\
& \leq C \sum_{\nu}\left(\left\|\tilde{\zeta}_{\nu} \Psi_{\nu, A}^{+} \zeta_{\nu} \varphi^{\nu}\right\|_{\lambda^{+}}^{2}+\left\|\tilde{\zeta}_{\nu} \Psi_{\nu, A}^{0} \zeta_{\nu} \varphi^{\nu}\right\|_{0}^{2}+\left\|\tilde{\zeta}_{\nu} \Psi_{\nu, A}^{-} \zeta_{\nu} \varphi^{\nu}\right\|_{\lambda^{-}}^{2}\right)
\end{aligned}
$$

since $\lambda^{+}$and $\lambda^{-}$are positive, bounded, and bounded away from zero.

The meaning of Lemma 3.5 is that $\|\varphi\|_{ \pm} \sim\|\varphi\|_{0}^{2}$ with constants independent of $A$, so the Riesz Representation Theorem implies the following corollary (see Corollary 4.6 in [Nic06]).

Corollary 3.6. There exists a self-adjoint operator $E_{\lambda^{+}, \lambda^{-}}=E_{ \pm}$so that

$$
(\varphi, \phi)_{0}=\left\langle\varphi, E_{ \pm} \phi\right\rangle_{ \pm}
$$


for any two forms $\varphi$ and $\phi$ in $L^{2}(M) . E_{ \pm}$is the inverse of

$$
F_{ \pm}=\sum_{\nu}\left(\zeta_{\nu}\left(\Psi_{\nu, A}^{+}\right)^{*} \tilde{\zeta}_{\nu} e^{-\lambda^{+}} \tilde{\zeta}_{\nu} \Psi_{\nu, A}^{+} \zeta_{\nu}+\zeta_{\nu}\left(\Psi_{\nu, A}^{0}\right)^{*} \tilde{\zeta}_{\nu}^{2} \Psi_{\nu, A}^{0} \zeta_{\nu}+\zeta_{\nu}\left(\Psi_{\nu, A}^{-}\right)^{*} \tilde{\zeta}_{\nu} e^{\lambda^{-}} \tilde{\zeta}_{\nu} \Psi_{\nu, A}^{-} \tilde{\zeta}_{\nu}\right)
$$

$E_{ \pm}$and $F_{ \pm}$are bounded in $L^{2}(M)$ independently of $A \geq 1$ since $0 \leq \lambda^{+}, \lambda^{-} \leq 1$.

3.4. $\bar{\partial}_{b}$ and its adjoints. If $f$ is a function on $M$, in local coordinates,

$$
\bar{\partial}_{b} f=\sum_{j=1}^{n-1} \bar{L}_{j} f \bar{\omega}_{j}
$$

while if $\varphi$ is a $(0, q)$-form, there exist functions $m_{K}^{J}$ so that

$$
\bar{\partial}_{b} \varphi=\sum_{\substack{J \in \mathcal{I}^{\prime} \\ K \in \mathcal{I}_{q+1}^{\prime}}} \sum_{j=1}^{n-1} \epsilon_{K}^{j J} \bar{L}_{j} \varphi_{J} \bar{\omega}_{K}+\sum_{\substack{J \in \mathcal{I}^{\prime} \\ K \in \mathcal{I}_{q+1}^{\prime}}} \varphi_{J} m_{K}^{J} \bar{\omega}_{K} .
$$

Let $\bar{L}_{j}^{*}$ be the adjoint of $\bar{L}_{j}$ in $(\cdot, \cdot)_{0}, \bar{L}_{j}^{*++}$ be the adjoint of $\bar{L}_{j}$ in $(\cdot, \cdot)_{\lambda^{+}}$, and $\bar{L}_{j}^{*-}$ be the adjoint of $\bar{L}_{j}$ in $(\cdot, \cdot)_{\lambda^{-}}$. Then we define $\bar{\partial}_{b}^{*}, \bar{\partial}_{b}^{*,+}$, and $\bar{\partial}_{b}^{*,-}$ to be the adjoints of $\bar{\partial}_{b}$ in $L^{2}(M)$, $L^{2}\left(M, e^{-\lambda^{+}}\right)$, and $L^{2}\left(M, e^{\lambda^{-}}\right)$, respectively. On a $(0, q)$-form $\varphi$, we have (for some functions $\left.f_{j} \in C^{\infty}(U)\right)$

$$
\begin{gathered}
\bar{\partial}_{b}^{*} \varphi=\sum_{\substack{I \in \mathcal{I}_{q-1}^{\prime} \\
J \in \mathcal{I}_{q}^{\prime}}} \sum_{j=1}^{n-1} \epsilon_{J}^{j I} \bar{L}_{j}^{*} \varphi_{J} \bar{\omega}_{I}+\sum_{\substack{I \in \mathcal{I}_{q-1}^{\prime} \\
J \in \mathcal{I}_{q}^{\prime}}} \overline{m_{J}^{I}} \varphi_{J} \bar{\omega}_{I} \\
=-\sum_{\substack{I \in \mathcal{I}_{q}^{\prime} \\
J \in \mathcal{I}_{q}^{\prime}}} \sum_{j=1}^{n-1} \epsilon_{J}^{j I}\left(L_{j} \varphi_{J}+f_{j} \varphi_{J}\right) \bar{\omega}_{I}+\sum_{\substack{I \in \mathcal{I}_{q-1}^{\prime} \\
J \in \mathcal{I}_{q}^{\prime}}} \overline{m_{J}^{I}} \varphi_{J} \bar{\omega}_{I} \\
\bar{\partial}_{b}^{*,+} \varphi=\sum_{\substack{I \in \mathcal{I}_{q-1}^{\prime} \\
J \in \mathcal{I}_{q}^{\prime}}} \sum_{j=1}^{n-1} \epsilon_{J}^{j I} \bar{L}_{j}^{*,+} \varphi_{J} \bar{\omega}_{I}+\sum_{\substack{I \in \mathcal{I}_{q-1}^{\prime} \\
J \in \mathcal{I}_{q}^{\prime}}} \overline{m_{J}^{I}} \varphi_{J} \bar{\omega}_{I} \\
=-\sum_{\substack{I \in \mathcal{I}_{q-1}^{\prime} \\
J \in \mathcal{I}_{q}^{\prime}}} \sum_{j=1}^{n-1} \epsilon_{J}^{j I}\left(L_{j} \varphi_{J}-L_{j} \lambda^{+} \varphi_{J}+f_{j} \varphi_{J}\right) \bar{\omega}_{I}+\sum_{\substack{I \in \mathcal{I}_{q}^{\prime}-1 \\
J \in \mathcal{I}_{q}^{\prime}}} \overline{m_{J}^{I}} \varphi_{J} \bar{\omega}_{I}
\end{gathered}
$$




$$
\begin{aligned}
\bar{\partial}_{b}^{*,-} \varphi & =\sum_{\substack{I \in \mathcal{I}_{q-1}^{\prime} \\
J \in \mathcal{I}_{q}^{\prime}}} \sum_{j=1}^{n-1} \epsilon_{J}^{j I} \bar{L}_{j}^{*,-} \varphi_{J} \bar{\omega}_{I}+\sum_{\substack{I \in \mathcal{I}_{q-1}^{\prime} \\
J \in \mathcal{I}_{q}^{\prime}}} \bar{m}_{J}^{I} \varphi_{J} \bar{\omega}_{I} \\
& =-\sum_{\substack{I \in \mathcal{I}_{q-1}^{\prime} \\
J \in \mathcal{I}_{q}^{\prime}}} \sum_{j=1}^{n-1} \epsilon_{J}^{j I}\left(L_{j} \varphi_{J}+L_{j} \lambda^{-} \varphi_{J}+f_{j} \varphi_{J}\right) \bar{\omega}_{I}+\sum_{\substack{I \in \mathcal{I}_{q-1}^{\prime} \\
J \in \mathcal{I}_{q}^{\prime}}} \overline{m_{J}^{I}} \varphi_{J} \bar{\omega}_{I}
\end{aligned}
$$

Consequently, we see that

$$
\bar{\partial}_{b}^{*,+}=\bar{\partial}_{b}^{*}-\left[\bar{\partial}_{b}^{*}, \lambda^{+}\right] \text {and } \bar{\partial}_{b}^{*,-}=\bar{\partial}_{b}^{*}+\left[\bar{\partial}_{b}^{*}, \lambda^{-}\right]
$$

and all three adjoints have the same domain. Finally, let $\bar{\partial}_{b, \pm}^{*}$ be the adjoint of $\bar{\partial}_{b}$ with respect to $\langle\cdot, \cdot\rangle_{ \pm}$.

The computations proving Lemma 4.8 and Lemma 4.9 and equation (4.4) in [Nic06] can be applied here with only a change of notation, so we have the following two results, recorded here as Lemma 3.7 and Lemma 3.8. The meaning of the results is that $\bar{\partial}_{b, \pm}^{*}$ acts like $\bar{\partial}_{b}^{*,+}$ for forms whose support is basically $\mathcal{C}^{+}$and $\bar{\partial}_{b}^{*,-}$ on forms whose support is basically $\mathcal{C}^{-}$.

Lemma 3.7. On smooth $(0, q)$-forms,

$$
\begin{aligned}
\bar{\partial}_{b, \pm}^{*}=\bar{\partial}_{b}^{*}- & \sum_{\mu} \zeta_{\mu}^{2} \tilde{\Psi}_{\mu, A}^{+}\left[\bar{\partial}_{b}^{*}, \lambda^{+}\right]+\sum_{\mu} \zeta_{\mu}^{2} \tilde{\Psi}_{\mu, A}^{-}\left[\bar{\partial}_{b}^{*}, \lambda^{-}\right] \\
& +\sum_{\mu}\left(\tilde{\zeta}_{\mu}\left[\tilde{\zeta}_{\mu} \Psi_{\mu, A}^{+} \zeta_{\mu}, \bar{\partial}_{b}\right]^{*} \tilde{\zeta}_{\mu} \Psi_{\mu, A}^{+} \zeta_{\mu}+\zeta_{\mu}\left(\Psi_{\mu, A}^{+}\right)^{*} \tilde{\zeta}_{\mu}\left[\bar{\partial}_{b}^{*,+}, \tilde{\zeta}_{\mu} \Psi_{\mu, A}^{+} \zeta_{\mu}\right] \tilde{\zeta}_{\mu}\right. \\
& \left.\quad+\tilde{\zeta}_{\mu}\left[\tilde{\zeta}_{\mu} \Psi_{\mu, A}^{-} \zeta_{\mu}, \bar{\partial}_{b}\right]^{*} \tilde{\zeta}_{\mu} \Psi_{\mu, A}^{-} \zeta_{\mu}+\zeta_{\mu}\left(\Psi_{\mu, A}^{+}\right)^{*} \tilde{\zeta}_{\mu}\left[\bar{\partial}_{b}^{*,-}, \tilde{\zeta}_{\mu} \Psi_{\mu, A}^{-} \zeta_{\mu}\right] \tilde{\zeta}_{\mu}+E_{A}\right),
\end{aligned}
$$

where the error term $E_{A}$ is a sum of order zero terms and lower order terms. Also, the symbol of $E_{A}$ is supported in $\mathcal{C}_{\mu}^{0}$ for each $\mu$.

We are now ready to define the energy forms that we use. Let

$$
\begin{aligned}
Q_{b, \pm}(\phi, \varphi) & =\left\langle\bar{\partial}_{b} \phi, \bar{\partial}_{b} \varphi\right\rangle_{ \pm}+\left\langle\bar{\partial}_{b, \pm}^{*} \phi, \bar{\partial}_{b, \pm}^{*} \varphi\right\rangle_{ \pm} \\
Q_{b,+}(\phi, \varphi) & =\left(\bar{\partial}_{b} \phi, \bar{\partial}_{b} \varphi\right)_{\lambda^{+}}+\left(\bar{\partial}_{b}^{*,+} \phi, \bar{\partial}_{b}^{*,+} \varphi\right)_{\lambda^{+}} \\
Q_{b, 0}(\phi, \varphi) & =\left(\bar{\partial}_{b} \phi, \bar{\partial}_{b} \varphi\right)_{0}+\left(\bar{\partial}_{b}^{*} \phi, \bar{\partial}_{b}^{*} \varphi\right)_{0} \\
Q_{b,-}(\phi, \varphi) & =\left(\bar{\partial}_{b} \phi, \bar{\partial}_{b} \varphi\right)_{\lambda^{-}}+\left(\bar{\partial}_{b}^{*,-} \phi, \bar{\partial}_{b}^{*,-} \varphi\right)_{\lambda^{-}}
\end{aligned}
$$

Lemma 3.8. If $\varphi$ is a smooth $(0, q)$-form on $M$, then there exist constants $K, K_{ \pm}, K^{\prime}$ with $K \geq 1$ so that

$$
\begin{array}{r}
K Q_{b, \pm}(\varphi, \varphi)+K_{ \pm} \sum_{\nu}\left\|\tilde{\zeta}_{\nu} \tilde{\Psi}_{\nu, A}^{0} \zeta_{\nu} \varphi^{\nu}\right\|_{0}^{2}+K^{\prime}\|\varphi\|_{0}^{2}+O_{ \pm}\left(\|\varphi\|_{-1}^{2}\right) \geq \sum_{\nu}\left[Q_{b,+}\left(\tilde{\zeta}_{\nu} \Psi_{\nu, A}^{+} \zeta_{\nu} \varphi^{\nu}, \tilde{\zeta}_{\nu} \Psi_{\nu, A}^{+} \zeta_{\nu} \varphi^{\nu}\right)\right. \\
\left.+Q_{b, 0}\left(\tilde{\zeta}_{\nu} \Psi_{\nu, A}^{0} \zeta_{\nu} \varphi^{\nu}, \tilde{\zeta}_{\nu} \Psi_{\nu, A}^{0} \zeta_{\nu} \varphi^{\nu}\right)+Q_{b,-}\left(\tilde{\zeta}_{\nu} \Psi_{\nu, A}^{-} \zeta_{\nu} \varphi^{\nu}, \tilde{\zeta}_{\nu} \Psi_{\nu, A}^{-} \zeta_{\nu} \varphi^{\nu}\right)\right]
\end{array}
$$


$K$ and $K^{\prime}$ do not depend on $A$.

Many of the subsequent proofs make use of the "lc/sc" argument: $-\epsilon\|x\|^{2}-\epsilon^{-1}\|y\|^{2} \leq$ $2 \operatorname{Re}((x, y)) \leq \epsilon\|x\|^{2}+\epsilon^{-1}\|y\|^{2}$ where $(\cdot, \cdot)$ is any Hermitian inner product with associated norm $\|\cdot\|$. Also, since that $\bar{\partial}_{b}^{*,+}=\bar{\partial}_{b}+$ lowerorder, commuting $\bar{\partial}_{b}^{*,+}$ by $\Psi_{\nu, A}^{+}$creates error terms of order 0 that do not depend on $\lambda^{+}$and lower order terms that may depend on $\lambda^{+}$.

\section{The Basic Estimate}

The goal of this section is to prove a basic estimate for smooth forms on $M$.

Proposition 4.1. Let $M \subset \mathbb{C}^{N}$ be a compact, orientable, weakly pseudoconvex $C R$-manifold of dimension $n \geq 5$ and $1 \leq q \leq n-2$. Assume that $M$ admits functions $\lambda^{+}$and $\lambda^{-}$where $\lambda^{+}$is strictly CR-plurisubharmonic on $(0, q)$-forms and $\lambda^{-}$is strictly CR-plurisubharmonic on $(0, n-1-q)$-forms Let $\varphi \in \operatorname{Dom}\left(\bar{\partial}_{b}\right) \cap \operatorname{Dom}\left(\bar{\partial}_{b}^{*}\right)$. There exist constants $K, K_{ \pm}$, and $K_{ \pm}^{\prime}$ where $K$ does not depend on $\lambda^{+}$and $\lambda^{-}$(and consequently $A$ ) so that

$$
A_{ \pm}\|\varphi\|_{ \pm}^{2} \leq K Q_{b, \pm}(\varphi, \varphi)+K\|\varphi\|_{ \pm}^{2}+K_{ \pm} \sum_{\nu} \sum_{J \in \mathcal{I}_{q}^{\prime}}\left\|\tilde{\zeta}_{\nu} \tilde{\Psi}_{\nu, A}^{0} \zeta_{\nu} \varphi_{J}^{\nu}\right\|_{0}^{2}+K_{ \pm}^{\prime}\|\varphi\|_{-1}^{2} .
$$

The constant $A_{ \pm}>0$ is the minimum of the CR-plurisubharmonicity constants $A_{\lambda^{+}}$and $A_{\lambda^{-}}$.

The proof of Proposition 4.1 comes as the culmination of a series of calculations that started with Lemma 3.8 .

4.1. Local Estimates. We work on a fixed $U=U_{\nu}$. On this neighborhood, as above, there exists an orthonormal basis of vector fields $L_{1}, \ldots, L_{n}, \bar{L}_{1}, \ldots, \bar{L}_{n}$ so that

$$
\left[L_{j}, \bar{L}_{k}\right]=c_{j k} T+\sum_{\ell=1}^{n-1}\left(d_{j k}^{\ell} L_{\ell}-\bar{d}_{k j}^{\ell} \bar{L}_{\ell}\right)
$$

if $1 \leq j, k \leq n-1$, and $T=L_{n}-\bar{L}_{n}$, and for some fixed point $P$,

$$
\left.\left[L_{j}, \bar{L}_{k}\right]\right|_{P}=c_{j k} T \text {. }
$$

Note that $c_{j k}$ are the coefficients of the Levi form. Recall that $\bar{L}^{*,+}, \bar{L}^{*}$, and $\bar{L}^{*,-}$ are the adjoints of $\bar{L}$ in $(\cdot, \cdot)_{\lambda^{+}},(\cdot, \cdot)_{0}$, and $(\cdot, \cdot)_{\lambda^{-}}$, respectively. From (3) , we see that

$$
\bar{L}_{j}^{*,+}=-L_{j}+L_{j}\left(\lambda^{+}\right)-f_{j} \quad \text { and } \quad \bar{L}_{j}^{*,-}=-L_{j}-L_{j}\left(\lambda^{-}\right)-f_{j},
$$

and plugging this into (5), we have

$$
\begin{aligned}
& {\left[\bar{L}_{j}^{*,+}, \bar{L}_{k}\right]=-c_{j k} T-\sum_{\ell=1}^{n-1}\left(d_{j k}^{\ell} L_{\ell}-\bar{d}_{k j}^{\ell} \bar{L}_{\ell}\right)-\bar{L}_{k} L_{j} \lambda^{+}+\bar{L}_{k} f_{j}} \\
& {\left[\bar{L}_{j}^{*,-}, \bar{L}_{k}\right]=-c_{j k} T-\sum_{k=1}^{n-1}\left(d_{j k}^{\ell} L_{\ell}-\bar{d}_{k j}^{\ell} \bar{L}_{k}\right)+\bar{L}_{k} L_{j} \lambda^{-}+\bar{L}_{k} f_{j}}
\end{aligned}
$$


For the inner product $Q_{b,+}(\varphi, \varphi)$, we have the following estimate.

Lemma 4.2. Let $\varphi$ be a $(0, q)$-form supported in $U, \varphi \in \operatorname{Dom}\left(\bar{\partial}_{b}\right) \cap \operatorname{Dom}\left(\bar{\partial}_{b}^{*}\right)$. There exists $0<\epsilon^{\prime} \ll 1$ so that

$$
\begin{aligned}
& Q_{b,+}(\varphi, \varphi) \geq\left(1-\epsilon^{\prime}\right) \sum_{J \in \mathcal{I}_{q}^{\prime}} \sum_{j=1}^{n-1}\left\|\bar{L}_{j} \varphi_{J}\right\|_{\lambda^{+}}^{2}+\sum_{J \in \mathcal{I}_{q}^{\prime}} \sum_{j \in J}\left[\operatorname{Re}\left\{\left(c_{j j} T \varphi_{J}, \varphi_{J}\right)_{\lambda^{+}}\right\}\right. \\
& \left.\left.+\frac{1}{2}\left(\left(\bar{L}_{j} L_{j}\left(\lambda^{+}\right)+L_{j} \bar{L}_{j}\left(\lambda^{+}\right)\right) \varphi_{J}, \varphi_{J}\right)_{\lambda^{+}}\right)+\frac{1}{2} \sum_{\ell=1}^{n-1}\left(\left(d_{j j}^{\ell} L_{\ell}\left(\lambda^{+}\right)+\bar{d}_{j j}^{\ell} \bar{L}_{\ell}\left(\lambda^{+}\right)\right) \varphi_{J}, \varphi_{J}\right)_{\lambda^{+}}\right] \\
& -\sum_{J, J^{\prime} \in \mathcal{I}_{q}^{\prime}} \sum_{\substack{1 \leq j, k \leq n-1 \\
j \neq k}} \epsilon_{j J^{\prime}}^{k J}\left[\operatorname{Re}\left\{\left(c_{j k} T \varphi_{J}, \varphi_{J^{\prime}}\right)_{\lambda^{+}}\right\}+\frac{1}{2}\left(\left(\bar{L}_{k} L_{j}\left(\lambda^{+}\right)+L_{j} \bar{L}_{k}\left(\lambda^{+}\right)\right) \varphi_{J}, \varphi_{J^{\prime}}\right)_{\lambda^{+}}\right) \\
& \left.+\frac{1}{2} \sum_{\ell=1}^{n-1}\left(\left(d_{j k}^{\ell} L_{\ell}\left(\lambda^{+}\right)+\bar{d}_{k j}^{\ell} \bar{L}_{\ell}\left(\lambda^{+}\right)\right) \varphi_{J}, \varphi_{J^{\prime}}\right)_{\lambda^{+}}\right]+O\left(\|\varphi\|_{0}^{2}\right) .
\end{aligned}
$$

Proof. First, observe

$$
\left(\bar{\partial}_{b}^{*,+} \varphi, \bar{\partial}_{b}^{*,+} \varphi\right)_{\lambda^{+}}=\sum_{\substack{I \in \mathcal{I}_{q-1}^{\prime} \\ J, J^{\prime} \in \mathcal{I}_{q}^{\prime}}} \sum_{\substack{1 \leq j, k \leq n-1 \\ j \neq k}} \epsilon_{J}^{j I} \epsilon_{J^{\prime}}^{k I}\left(\bar{L}_{j}^{*,+} \varphi_{J}, \bar{L}_{k}^{*,+} \varphi_{J^{\prime}}\right)_{\lambda^{+}}+O\left(\|\varphi\|_{\lambda^{+}}^{2}+\left(\sum_{j=1}^{n-1}\left\|\bar{L}_{j} \varphi\right\|_{\lambda^{+}}^{2}\right)^{1 / 2}\|\varphi\|_{\lambda^{+}}\right)
$$

However, if $j \neq k$, then $\epsilon_{J}^{j I} \epsilon_{J^{\prime}}^{k I}=\epsilon_{k J}^{k j I} \epsilon_{j J^{\prime}}^{j k I}=-\epsilon_{k J}^{j k I} \epsilon_{j J^{\prime}}^{j k I}=-\epsilon_{j J^{\prime}}^{k J}$. Consequently,

$$
\begin{aligned}
\left\|\bar{\partial}_{b}^{*,+} \varphi\right\|_{\lambda^{+}}^{2}= & \sum_{J \in \mathcal{I}_{q}^{\prime}} \sum_{j \in J}\left\|\bar{L}_{j}^{*,+} \varphi_{J}\right\|_{\lambda^{+}}^{2}-\sum_{J, J^{\prime} \in \mathcal{I}_{q}^{\prime}} \sum_{\substack{1 \leq j, k \leq n-1 \\
j \neq k}} \epsilon_{j J^{\prime}}^{k J}\left(\bar{L}_{j}^{*,+} \varphi_{J}, \bar{L}_{k}^{*,+} \varphi_{J^{\prime}}\right)_{\lambda^{+}} \\
& +O\left(\|\varphi\|_{\lambda^{+}}^{2}+\left(\sum_{j=1}^{n-1}\left\|\bar{L}_{j} \varphi\right\|_{\lambda^{+}}^{2}\right)^{1 / 2}\|\varphi\|_{\lambda^{+}}\right) \\
= & \sum_{J \in \mathcal{I}_{q}^{\prime}} \sum_{j \in J}\left\|\bar{L}_{j} \varphi_{J}\right\|_{\lambda^{+}}^{2}+\sum_{J \in \mathcal{I}_{q}^{\prime}} \sum_{j \in J}\left(\left[\bar{L}_{j}, \bar{L}_{j}^{*,+}\right] \varphi_{J}, \varphi_{J}\right)_{\lambda^{+}} \\
& -\sum_{J, J^{\prime} \in \mathcal{I}_{q}^{\prime}} \sum_{\substack{1 \leq j, k \leq n-1 \\
j \neq k}} \epsilon_{j J^{\prime}}^{k J}\left(\bar{L}_{j}^{*,+} \varphi_{J}, \bar{L}_{k}^{*,+} \varphi_{J^{\prime}}\right)_{\lambda^{+}}+O\left(\|\varphi\|_{\lambda^{+}}^{2}+\left(\sum_{j=1}^{n-1}\left\|\bar{L}_{j} \varphi\right\|_{\lambda^{+}}^{2}\right)^{1 / 2}\|\varphi\|_{\lambda^{+}}\right)
\end{aligned}
$$


Second, from the calculation of $\bar{\partial}_{b}$ above, we compute

$$
\begin{aligned}
& \left\|\bar{\partial}_{b} \varphi\right\|_{\lambda^{+}}^{2}=\sum_{\substack{J, J^{\prime} \in \mathcal{I}_{q}^{\prime} \\
K \in \mathcal{I}_{q+1}^{\prime}}} \sum_{\substack{1 \leq j, k \leq n-1 \\
j \neq k}} \epsilon_{K}^{k J} \epsilon_{K}^{j J^{\prime}}\left(\bar{L}_{k} \varphi_{J}, \bar{L}_{j} \varphi_{J^{\prime}}\right)_{\lambda^{+}} O\left(\|\varphi\|_{\lambda^{+}}^{2}+\left(\sum_{j=1}^{n-1}\left\|\bar{L}_{j} \varphi\right\|_{\lambda^{+}}^{2}\right)^{1 / 2}\|\varphi\|_{\lambda^{+}}\right) \\
& =\sum_{J \in \mathcal{I}_{q}} \sum_{j \notin J}\left\|\bar{L}_{j} \varphi_{J}\right\|_{\lambda^{+}}^{2}+\sum_{J, J^{\prime} \in \mathcal{I}_{q}} \sum_{\substack{1 \leq j, k \leq n-1 \\
j \neq k}} \epsilon_{j J^{\prime}}^{k J}\left(\bar{L}_{k} \varphi_{J}, \bar{L}_{j} \varphi_{J^{\prime}}\right)_{\lambda^{+}}+O\left(\|\varphi\|_{\lambda^{+}}^{2}+\left(\sum_{j=1}^{n-1}\left\|\bar{L}_{j} \varphi\right\|_{\lambda^{+}}^{2}\right)^{1 / 2}\|\varphi\|_{\lambda^{+}}\right) \\
& =\sum_{J \in \mathcal{I}_{q}} \sum_{j \notin J}\left\|\bar{L}_{j} \varphi_{J}\right\|_{\lambda^{+}}^{2}+\sum_{J, J^{\prime} \in \mathcal{I}_{q}} \sum_{\substack{1 \leq j, k \leq n-1 \\
j \neq k}} \epsilon_{j J^{\prime}}^{k J}\left(\bar{L}_{j}^{*,+} \varphi_{J}, \bar{L}_{k}^{*,+} \varphi_{J^{\prime}}\right)_{\lambda^{+}} \\
& +\sum_{J, J^{\prime} \in \mathcal{I}_{q}} \sum_{\substack{1 \leq j, k \leq n-1 \\
j \neq k}} \epsilon_{j J^{\prime}}^{k J}\left(\left[\bar{L}_{j}^{*,+}, \bar{L}_{k}\right] \varphi_{J}, \varphi_{J^{\prime}}\right)_{\lambda^{+}}+O\left(\|\varphi\|_{\lambda^{+}}^{2}+\left(\sum_{j=1}^{n-1}\left\|\bar{L}_{j} \varphi\right\|_{\lambda^{+}}^{2}\right)^{1 / 2}\|\varphi\|_{\lambda^{+}}\right) .
\end{aligned}
$$

By a lc/sc argument,

$$
\left(\sum_{j=1}^{n-1}\left\|\bar{L}_{j} \varphi\right\|_{\lambda^{+}}^{2}\right)^{1 / 2}\|\varphi\|_{\lambda^{+}} \geq-\epsilon \sum_{j=1}^{n-1}\left\|\bar{L}_{j} \varphi\right\|_{\lambda^{+}}^{2}-\frac{1}{\epsilon}\|\varphi\|_{\lambda^{+}}^{2},
$$

so adding together our computations yields

$$
\begin{aligned}
Q_{b,+}(\varphi, \varphi) \geq(1-\epsilon) \sum_{J \in \mathcal{I}_{q}^{\prime}} \sum_{j=1}^{n-1}\left\|\bar{L}_{j} \varphi_{J}\right\|_{\lambda^{+}}^{2}+\sum_{J \in \mathcal{I}_{q}} \sum_{j \in J}\left(\left[\bar{L}_{j}, \bar{L}_{j}^{*++}\right] \varphi_{J}, \varphi_{J}\right)_{\lambda^{+}} \\
+\sum_{J, J^{\prime} \in \mathcal{I}_{q}^{\prime}} \sum_{\substack{1 \leq j, k \leq n-1 \\
j \neq k}} \epsilon_{j J^{\prime}}^{k J}\left(\left[\bar{L}_{j}^{*,+}, \bar{L}_{k}\right] \varphi_{J}, \varphi_{J^{\prime}}\right)_{\lambda^{+}}+O\left(\|\varphi\|_{\lambda^{+}}^{2}\right) .
\end{aligned}
$$

Recall that the commutator

$$
\left[\bar{L}_{j}^{*++}, \bar{L}_{k}\right]=-c_{j k} T-\sum_{\ell=1}^{n-1}\left(d_{j k}^{\ell} L_{\ell}-\bar{d}_{k j}^{\ell} \bar{L}_{\ell}\right)-\bar{L}_{k} L_{j} \lambda^{+}+\bar{L}_{k} f_{j}
$$

and note that

$$
\left|\left(\bar{d}_{k j}^{\ell} \bar{L}_{\ell} \varphi_{J}, \varphi_{J^{\prime}}\right)_{\lambda^{+}}\right| \leq \epsilon\left\|\bar{L}_{\ell} \varphi_{J}\right\|_{\lambda^{+}}^{2}+C_{\epsilon}\|\varphi\|_{\lambda^{+}}^{2} .
$$


Consequently,

$$
\begin{aligned}
& Q_{b,+}(\varphi, \varphi) \geq(1-\epsilon) \sum_{J \in \mathcal{I}_{q}^{\prime}} \sum_{j=1}^{n-1}\left\|\bar{L}_{j} \varphi_{J}\right\|_{\lambda^{+}}^{2} \\
& \quad+\operatorname{Re}\left\{\sum_{J \in \mathcal{I}_{q}^{\prime}} \sum_{j \in J}\left[\left(c_{j j} T \varphi_{J}, \varphi_{J}\right)_{\lambda^{+}}+\sum_{\ell=1}^{n-1}\left(d_{j j}^{\ell} L_{\ell} \varphi_{J}, \varphi_{J}\right)_{\lambda^{+}}+\left(\bar{L}_{j} \bar{L}_{j} \lambda^{+} \varphi_{J}, \varphi_{J}\right)_{\lambda^{+}}\right]\right\} \\
& -\operatorname{Re}\left\{\sum_{J, J^{\prime} \in \mathcal{I}_{q}^{\prime}} \sum_{\substack{1 \leq j, k \leq n-1 \\
j \neq k}} \epsilon_{j J^{\prime}}^{k J}\left[\left(c_{j k} T \varphi_{J}, \varphi_{J^{\prime}}\right)_{\lambda^{+}}+\sum_{\ell=1}^{n-1}\left(d_{j k}^{\ell} L_{\ell} \varphi_{J}, \varphi_{J^{\prime}}\right)_{\lambda^{+}}+\left(\bar{L}_{k} L_{j} \lambda^{+} \varphi_{J}, \varphi_{J^{\prime}}\right)_{\lambda^{+}}\right]\right\}+O\left(\|\varphi\|_{0}^{2}\right) .
\end{aligned}
$$

Also,

$$
\begin{array}{r}
\epsilon_{j J^{\prime}}^{k J} \operatorname{Re}\left\{\left(d_{j k}^{\ell} L_{\ell} \varphi_{J}, \varphi_{J^{\prime}}\right)_{\lambda^{+}}\right\}=\epsilon_{j J^{\prime}}^{k J} \operatorname{Re}\left\{\left(L_{\ell}\left(d_{j k}^{\ell} \varphi_{J}\right), \varphi_{J^{\prime}}\right)_{\lambda^{+}}-\epsilon_{j J^{\prime}}^{k J} \operatorname{Re}\left\{\left(L_{\ell}\left(d_{j k}^{\ell}\right) \varphi_{J}, \varphi_{J^{\prime}}\right)_{\lambda^{+}}\right\}\right. \\
=\epsilon_{j J^{\prime}}^{k J} \operatorname{Re}\left\{\left(-\bar{L}_{\ell}^{*,+}\left(d_{j k}^{\ell} \varphi_{J}\right), \varphi_{J^{\prime}}\right)_{\lambda^{+}}+\left(d_{j k}^{\ell} L_{\ell}\left(\lambda^{+}\right) \varphi_{J}, \varphi_{J^{\prime}}\right)_{\lambda^{+}}\right\}+O\left(\|\varphi\|_{\lambda^{+}}^{2}\right) \\
\geq-\epsilon\left\|\bar{L}_{\ell} \varphi_{J^{\prime}}\right\|_{\lambda^{+}}^{2}+\epsilon_{j J^{\prime}}^{k J} \operatorname{Re}\left\{\left(d_{j k}^{\ell} L_{\ell}\left(\lambda^{+}\right) \varphi_{j}, \varphi_{k}\right)_{\lambda^{+}}\right\}+O\left(\|\varphi\|_{\lambda^{+}}^{2}\right) .
\end{array}
$$

Recalling that $\operatorname{Re} z=\operatorname{Re} \bar{z}$ for any complex number $z$, we have

$$
\begin{aligned}
& \sum_{J, J^{\prime} \in \mathcal{I}_{q}} \sum_{j, k, \ell=1}^{n-1} \epsilon_{j J^{\prime}}^{k J} \operatorname{Re}\left\{\left(d_{j k}^{\ell} L_{\ell}\left(\lambda^{+}\right) \varphi_{J}, \varphi_{J^{\prime}}\right)_{\lambda^{+}}\right\} \\
= & \frac{1}{2} \sum_{J, J^{\prime} \in \mathcal{I}_{q}^{\prime}} \sum_{j, k, \ell=1}^{n-1} \epsilon_{j J^{\prime}}^{k J} \operatorname{Re}\left\{\left(d_{j k}^{\ell} L_{\ell}\left(\lambda^{+}\right) \varphi_{J}, \varphi_{J^{\prime}}\right)_{\lambda^{+}}+\left(d_{k j}^{\ell} L_{\ell}\left(\lambda^{+}\right) \varphi_{J^{\prime}}, \varphi_{J}\right)_{\lambda^{+}}\right\} \\
= & \frac{1}{2} \sum_{J, J^{\prime} \in \mathcal{I}_{q}^{\prime}} \sum_{j, k, \ell=1}^{n-1} \epsilon_{j J^{\prime}}^{k J} \operatorname{Re}\left\{\left(d_{j k}^{\ell} L_{\ell}\left(\lambda^{+}\right) \varphi_{J}, \varphi_{J^{\prime}}\right)_{\lambda^{+}}+\left(\varphi_{J^{\prime}}, \bar{d}_{k j}^{\ell} \bar{L}_{\ell}\left(\lambda^{+}\right) \varphi_{J}\right)_{\lambda^{+}}\right\} \\
= & \frac{1}{2} \sum_{J, J^{\prime} \in \mathcal{I}_{q}^{\prime}} \sum_{j, k, \ell=1}^{n-1} \epsilon_{j J^{\prime}}^{k J} \operatorname{Re}\left\{\left(d_{j k}^{\ell} L_{\ell}\left(\lambda^{+}\right) \varphi_{J}, \varphi_{J^{\prime}}\right)_{\lambda^{+}}+\overline{\left(\bar{d}_{k j}^{\ell} \bar{L}_{\ell}\left(\lambda^{+}\right) \varphi_{J}, \varphi_{J^{\prime}}\right)_{\lambda^{+}}}\right\} \\
= & \frac{1}{2} \sum_{J, J^{\prime} \in \mathcal{I}_{q}^{\prime}} \sum_{j, k, \ell=1}^{n-1} \epsilon_{j J^{\prime}}^{k J}\left(\left(d_{j k}^{\ell} L_{\ell}\left(\lambda^{+}\right) \varphi_{J}, \varphi_{J^{\prime}}\right)_{\lambda^{+}}+\left(\bar{d}_{k j}^{\ell} \bar{L}_{\ell}\left(\lambda^{+}\right) \varphi_{J}, \varphi_{J^{\prime}}\right)_{\lambda^{+}}\right)
\end{aligned}
$$

Similarly,

$$
\begin{aligned}
& \operatorname{Re}\left\{\sum_{J, J^{\prime} \in \mathcal{I}_{q}^{\prime}} \sum_{\substack{1 \leq j, k \leq n-1 \\
j \neq k}} \epsilon_{j J^{\prime}}^{k J}\left(\bar{L}_{k} L_{j} \lambda^{+} \varphi_{J}, \varphi_{J^{\prime}}\right)_{\lambda^{+}}\right\} \\
& =\frac{1}{2} \sum_{J, J^{\prime} \in \mathcal{I}_{q}^{\prime}} \sum_{\substack{1 \leq j, k \leq n-1 \\
j \neq k}} \epsilon_{j J^{\prime}}^{k J}\left(\left(\bar{L}_{k} L_{j} \lambda^{+} \varphi_{J}, \varphi_{J^{\prime}}\right)_{\lambda^{+}}+\left(L_{j} \bar{L}_{k} \lambda^{+} \varphi_{J}, \varphi_{J^{\prime}}\right)_{\lambda^{+}}\right)
\end{aligned}
$$


Next, we concentrate on the $Q_{b,-}(\varphi, \varphi)$ term.

Lemma 4.3. Let $\varphi$ be a $(0, q)$-form supported in $U, \varphi \in \operatorname{Dom}\left(\bar{\partial}_{b}\right) \cap \operatorname{Dom}\left(\bar{\partial}_{b}^{*}\right)$. There exists $0<\epsilon^{\prime} \ll 1$ so that

$$
\begin{aligned}
& Q_{b,-}(\varphi, \varphi) \geq\left(1-\epsilon^{\prime}\right) \sum_{J \in \mathcal{I}_{q}^{\prime}} \sum_{j=1}^{n-1}\left[\left\|\bar{L}_{j}^{*,-} \varphi_{J}\right\|_{\lambda^{-}}^{2}+\sum_{J \in \mathcal{I}_{q}^{\prime}} \sum_{j \in J}\left[\operatorname{Re}\left\{-\left(c_{j j} T \varphi_{J}, \varphi_{J}\right)_{\lambda^{-}}\right\}\right.\right. \\
& \left.\left.+\frac{1}{2}\left(\left(\bar{L}_{j} L_{j}\left(\lambda^{-}\right)+L_{j} \bar{L}_{j}\left(\lambda^{-}\right)\right) \varphi_{J}, \varphi_{J}\right)_{\lambda^{-}}\right)+\frac{1}{2} \sum_{\ell=1}^{n-1}\left(\left(d_{j j}^{\ell} L_{\ell}\left(\lambda^{-}\right)+\bar{d}_{j j}^{\ell} \bar{L}_{\ell}\left(\lambda^{-}\right)\right) \varphi_{J}, \varphi_{J}\right)_{\lambda^{-}}\right] \\
& +\sum_{J, J^{\prime} \in \mathcal{I}_{q}^{\prime}} \sum_{\substack{1 \leq j, k \leq n-1 \\
j \neq k}} \epsilon_{j J^{\prime}}^{k J}\left[\operatorname{Re}\left\{-\left(c_{j k} T \varphi_{J}, \varphi_{J^{\prime}}\right)_{\lambda^{-}}\right\}+\frac{1}{2}\left(\left(\bar{L}_{k} L_{j}\left(\lambda^{-}\right)+L_{j} \bar{L}_{k}\left(\lambda^{-}\right)\right) \varphi_{J}, \varphi_{J^{\prime}}\right)_{\lambda^{-}}\right) \\
& \left.+\frac{1}{2} \sum_{\ell=1}^{n-1}\left(\left(d_{j k}^{\ell} L_{\ell}\left(\lambda^{-}\right)+\bar{d}_{k j}^{\ell} \bar{L}_{\ell}\left(\lambda^{-}\right)\right) \varphi_{J}, \varphi_{J^{\prime}}\right)_{\lambda^{-}}\right]+O\left(\|\varphi\|_{0}^{2}\right) .
\end{aligned}
$$

Proof. This lemma is proved with the same techniques as the previous lemma. By the argument leading up to ([6]), we have

$$
\begin{aligned}
Q_{b,-}(\varphi, \varphi) & =\sum_{J \in \mathcal{I}_{q}^{\prime}} \sum_{j \notin J}\left\|\bar{L}_{j} \varphi_{J}\right\|_{\lambda^{-}}^{2}+\sum_{J, J^{\prime} \in \mathcal{I}_{q}} \sum_{\substack{1 \leq j, k \leq n-1 \\
j \neq k}} \epsilon_{j J^{\prime}}^{k J}\left(\bar{L}_{j}^{*,-} \varphi_{J}, \bar{L}_{k}^{*,-} \varphi_{J^{\prime}}\right)_{\lambda^{-}} \\
& +\sum_{J, J^{\prime} \in \mathcal{I}_{q}} \sum_{\substack{1 \leq j, k \leq n-1 \\
j \neq k}} \epsilon_{j J^{\prime}}^{k J}\left(\left[\bar{L}_{j}^{*,-}, \bar{L}_{k}\right] \varphi_{J}, \varphi_{J^{\prime}}\right)_{\lambda^{-}} \\
& +\sum_{J \in \mathcal{I}_{q}^{\prime}} \sum_{j \in J}\left\|\bar{L}_{j}^{*,-} \varphi_{J}\right\|_{\lambda^{-}}^{2}-\sum_{J, J^{\prime} \in \mathcal{I}_{q}^{\prime}} \sum_{\substack{1 \leq j, k \leq n-1 \\
j \neq k}} \epsilon_{j J^{\prime}}^{k J}\left(\bar{L}_{j}^{*,-} \varphi_{J}, \bar{L}_{k}^{*,-} \varphi_{J^{\prime}}\right)_{\lambda^{-}} \\
& +O\left(\|\varphi\|_{\lambda^{-}}^{2}+\left(\sum_{J \in \mathcal{I}_{q}^{\prime}} \sum_{j=1}^{n-1}\left\|\bar{L}_{j}^{*,-} \varphi_{J}\right\|_{\lambda^{-}}^{2}\right)^{1 / 2}\|\varphi\|_{\lambda^{-}}\right)
\end{aligned}
$$

By integration by parts,

$$
\left\|\bar{L}_{j} \varphi_{J}\right\|_{\lambda^{-}}^{2}=\left\|\bar{L}_{j}^{*,-} \varphi_{J}\right\|_{\lambda^{-}}^{2}+\left(\left[\bar{L}_{j}^{*,-}, \bar{L}_{j}\right] \varphi_{J}, \varphi_{J}\right)_{\lambda^{-}} .
$$

Thus,

$$
\begin{aligned}
& Q_{b,-}(\varphi, \varphi)=\sum_{J \in \mathcal{I}_{q}^{\prime}} \sum_{j=1}^{n-1}\left\|\bar{L}_{j}^{*,-} \varphi_{J}\right\|_{\lambda^{-}}^{2}+\sum_{J \in \mathcal{I}_{q}^{\prime}} \sum_{j \notin J}\left(\left[\bar{L}_{j}^{*,-}, \bar{L}_{j}\right] \varphi_{J}, \varphi_{J}\right)_{\lambda^{-}} \\
& +\sum_{J, J^{\prime} \in \mathcal{I}_{q}} \sum_{\substack{1 \leq j, k \leq n-1 \\
j \neq k}} \epsilon_{j J^{\prime}}^{k J}\left(\left[\bar{L}_{j}^{*,-}, \bar{L}_{k}\right] \varphi_{J}, \varphi_{J^{\prime}}\right)_{\lambda^{-}}+O\left(\|\varphi\|_{\lambda^{-}}^{2}+\left(\sum_{J \in \mathcal{I}_{q}^{\prime}} \sum_{j=1}^{n-1}\left\|\bar{L}_{j}^{*,-} \varphi_{J}\right\|_{\lambda^{-}}^{2}\right)^{1 / 2}\|\varphi\|_{\lambda^{-}}\right) .
\end{aligned}
$$

Following the argument of Lemma 4.2, we proceed as above. 
The significance of the estimates in Lemma 4.2 and Lemma 4.3 is demonstrated by the multilinear algebra in Appendix $\mathrm{A}$, and it highlights the need for $\left(\mathrm{CR}-P_{q}\right)$ as well as (CR$\left.P_{n-1-q}\right)$.

We need the following versions of the sharp Gårding inequality. This is Theorem 7.1 in [Nic06] written for forms. It can be proved by following proofs of Theorem 3.1 and Theorem 3.2 in [LN66] line by line (making the obvious modifications).

Theorem 4.4. If $P=\left(p_{j k}(z, D)\right)$ is a matrix first order pseudodifferential operator. If $p(z, \xi)$ is Hermitian and the sum of any collection of $q$ eigenvalues is nonnegative, then there exists a constant $C>0$ so that for any $(0, q)$-form $u$,

$$
\operatorname{Re}\left\{\sum_{J \in \mathcal{I}_{q}^{\prime}}\left(p_{j j}(\cdot, D) u_{J}, u_{J}\right)-\sum_{J, J^{\prime} \in \mathcal{I}_{q}^{\prime}} \sum_{\substack{1 \leq j, k \leq m \\ j \neq k}} \epsilon_{j J^{\prime}}^{k J}\left(p_{j k}(\cdot, D) u_{J}, u_{J^{\prime}}\right)\right\} \geq-C\|u\|^{2} .
$$

If $p(z, \xi)$ is Hermitian and the sum of any collection of $(n-1-q)$ eigenvalues is nonnegative, then

$$
\operatorname{Re}\left\{\sum_{J \in \mathcal{I}_{q}^{\prime}}\left(p_{j j}(\cdot, D) u_{J}, u_{J}\right)+\sum_{J, J^{\prime} \in \mathcal{I}_{q}^{\prime}} \sum_{\substack{1 \leq j, k \leq m \\ j \neq k}} \epsilon_{j J^{\prime}}^{k J}\left(p_{j k}(\cdot, D) u_{J}, u_{J^{\prime}}\right)\right\} \geq-C\|u\|^{2} .
$$

Corollary 4.5. Let $R$ be a first order pseudodifferential operator such that $\sigma(R) \geq \kappa$ where $\kappa$ is some positive constant and $\left(h_{j k}\right)$ a hermitian matrix (that does not depend on $\xi$ ). Then there exists a constant $C$ such that if the sum of any $q$ eigenvalue of $\left(h_{j k}\right)$ is nonnegative, then

$$
\begin{aligned}
\operatorname{Re}\left\{\sum_{J \in \mathcal{I}_{q}^{\prime}}\left(h_{j j} R u_{J}, u_{J}\right)\right. & \left.-\sum_{J, J^{\prime} \in \mathcal{I}_{q}^{\prime}} \sum_{\substack{1 \leq j, k \leq m \\
j \neq k}} \epsilon_{j J^{\prime}}^{k J}\left(h_{j k} R u_{J}, u_{J^{\prime}}\right)\right\} \\
& \geq \kappa \operatorname{Re}\left\{\sum_{J \in \mathcal{I}_{q}^{\prime}}\left(\left(h_{j j} u_{J}, u_{J}\right)-\sum_{J, J^{\prime} \in \mathcal{I}_{q}^{\prime}} \sum_{\substack{1 \leq j, k \leq m \\
j \neq k}} \epsilon_{j J^{\prime}}^{k J}\left(h_{j k} u_{J}, u_{J^{\prime}}\right)\right\}-C\|u\|^{2} .\right.
\end{aligned}
$$

and if the the sum of any collection of $(n-1-q)$ eigenvalues of $\left(h_{j k}\right)$ is nonnegative, then

$$
\begin{aligned}
\sum_{j k} \operatorname{Re}\left\{\sum_{J \in \mathcal{I}_{q}^{\prime}}\left(\left(h_{j j} R u_{J}, u_{J}\right)+\sum_{J, J^{\prime} \in \mathcal{I}_{q}^{\prime}} \sum_{\substack{1 \leq j, k \leq m \\
j \neq k}} \epsilon_{j J^{\prime}}^{k J}\left(h_{j k} R u_{J}, u_{J^{\prime}}\right)\right\}\right. \\
\geq \kappa \sum_{j k} \operatorname{Re}\left\{\sum_{J \in \mathcal{I}_{q}^{\prime}}\left(\left(h_{j j} u_{J}, u_{J}\right)+\sum_{J, J^{\prime} \in \mathcal{I}_{q}^{\prime}} \sum_{\substack{1 \leq j, k \leq m \\
j \neq k}} \epsilon_{j J^{\prime}}^{k J}\left(h_{j k} u_{J}, u_{J^{\prime}}\right)\right\}-C\|u\|^{2} .\right.
\end{aligned}
$$

Note that $\left(h_{j k}\right)$ may be a matrix-valued function in $z$ but may not depend on $\xi$.

Proof. Apply the previous theorem with $P$ where $p_{j k}=h_{j k}(R-\kappa)$.

We need Gårding's inequality to prove the following analog to Lemma 4.12 in [Nic06]. 
Lemma 4.6. Let $M$ be a weakly pseudoconvex $C R$-manifold and $\varphi$ a $(0, q)$-form supported on $U^{\prime}$ so that up to a smooth term $\hat{\varphi}$ is supported in $\mathcal{C}^{+}$. Then

$$
\begin{aligned}
& \operatorname{Re}\left\{\sum_{J \in \mathcal{I}_{q}^{\prime}}\left(c_{j j} T \varphi_{J}, \varphi_{J}\right)_{\lambda^{+}}-\sum_{J, J^{\prime} \in \mathcal{I}_{q}^{\prime}} \sum_{\substack{1 \leq j, k \leq m \\
j \neq k}} \epsilon_{j J^{\prime}}^{k J}\left(c_{j k} T \varphi_{J}, \varphi_{J^{\prime}}\right)_{\lambda^{+}}\right\} \\
& \geq A \operatorname{Re}\left\{\sum_{J \in \mathcal{I}_{q}^{\prime}}\left(\left(c_{j j} \varphi_{J}, \varphi_{J}\right)_{\lambda^{+}}-\sum_{J, J^{\prime} \in \mathcal{I}_{q}^{\prime}} \sum_{\substack{1 \leq j, k \leq m \\
j \neq k}} \epsilon_{j J^{\prime}}^{k J}\left(c_{j k} \varphi_{J}, \varphi_{J^{\prime}}\right)_{\lambda^{+}}\right\}+O\left(\|\varphi\|_{\lambda^{+}}^{2}\right)+O_{A}\left(\left\|\tilde{\zeta}_{\nu} \tilde{\Psi}_{A}^{0} \varphi\right\|_{0}^{2}\right) .\right.
\end{aligned}
$$

where the constant in $O\left(\|\varphi\|_{\lambda^{+}}^{2}\right)$ does not depend on $A$.

Proof. Let $\tilde{\Psi}_{A}^{+}$be a pseudodifferential operator of order zero whose symbol dominates $\hat{\phi}$ (up to a smooth error) and is supported in $\tilde{\mathcal{C}}^{+}$. By the support conditions of $\varphi$ and $\hat{\varphi}$,

$$
\begin{aligned}
& \sum_{J \in \mathcal{I}_{q}^{\prime}}\left(c_{j j} T \varphi_{J}, \varphi_{J}\right)_{\lambda^{+}}-\sum_{J, J^{\prime} \in \mathcal{I}_{q}^{\prime}} \sum_{\substack{1 \leq j, k \leq m \\
j \neq k}} \epsilon_{j J^{\prime}}^{k J}\left(c_{j k} T \varphi_{J}, \varphi_{J^{\prime}}\right)_{\lambda^{+}} \\
& =\sum_{J \in \mathcal{I}_{q}^{\prime}}\left(c_{j j} T \varphi_{J},\left(\left(\tilde{\Psi}_{A}^{+}\right)^{*} \tilde{\Psi}_{A}^{+}+\left(I d-\left(\tilde{\Psi}_{A}^{+}\right)^{*} \tilde{\Psi}_{A}^{+}\right)\right) \varphi_{J}\right)_{\lambda^{+}} \\
& -\sum_{J, J^{\prime} \in \mathcal{I}_{q}^{\prime}} \sum_{\substack{1 \leq j, k \leq m \\
j \neq k}} \epsilon_{j J^{\prime}}^{k J}\left(c_{j k} T \varphi_{J},\left(\left(\tilde{\Psi}_{A}^{+}\right)^{*} \tilde{\Psi}_{A}^{+}+\left(I d-\left(\tilde{\Psi}_{A}^{+}\right)^{*} \tilde{\Psi}_{A}^{+}\right)\right) \varphi_{J^{\prime}}\right)_{\lambda^{+}} \\
& =\sum_{J \in \mathcal{I}_{q}^{\prime}}\left(c_{j j} T \varphi_{J},\left(\tilde{\Psi}_{A}^{+}\right)^{*} \tilde{\Psi}_{A}^{+} \varphi_{J}\right)_{\lambda^{+}} \\
& -\sum_{J, J^{\prime} \in \mathcal{I}_{q}^{\prime}} \sum_{\substack{1 \leq j, k \leq m \\
j \neq k}} \epsilon_{j J^{\prime}}^{k J}\left(c_{j k} T \varphi_{J},\left(\tilde{\Psi}_{A}^{+}\right)^{*} \tilde{\Psi}_{A}^{+} \varphi_{J^{\prime}}\right)_{\lambda^{+}}+\text {smoother terms } \\
& =\sum_{J \in \mathcal{I}_{q}^{\prime}}\left(\tilde{\zeta} e^{-\lambda^{+}} c_{j j} \tilde{\Psi}_{A}^{+} T \varphi_{J}, \tilde{\zeta} \tilde{\Psi}_{A}^{+} \varphi_{J}\right)_{0} \\
& -\sum_{J, J^{\prime} \in \mathcal{I}_{q}^{\prime}} \sum_{\substack{1 \leq j, k \leq m \\
j \neq k}} \epsilon_{j J^{\prime}}^{k J}\left(\tilde{\zeta} e^{-\lambda^{+}} c_{j k} \tilde{\Psi}_{A}^{+} T \varphi_{J}, \tilde{\zeta} \tilde{\Psi}_{A}^{+} \varphi_{J^{\prime}}\right)_{0}+\text { smoother terms } \\
& =\sum_{J \in \mathcal{I}_{q}^{\prime}}\left(\tilde{\zeta}\left(\tilde{\Psi}_{A}^{+}\right)^{*} \tilde{\zeta}^{2} e^{-\lambda^{+}} c_{j j} \tilde{\Psi}_{A}^{+} T \varphi_{J}, \varphi_{J}\right)_{0} \\
& -\sum_{J, J^{\prime} \in \mathcal{I}_{q}^{\prime}} \sum_{\substack{1 \leq j, k \leq m \\
j \neq k}} \epsilon_{j J^{\prime}}^{k J}\left(\tilde{\zeta}\left(\tilde{\Psi}_{A}^{+}\right)^{*} \tilde{\zeta}^{2} e^{-\lambda^{+}} c_{j k} \tilde{\Psi}_{A}^{+} T \varphi_{J}, \varphi_{J^{\prime}}\right)_{0}+\text { smoother terms } \\
& =\sum_{j, k=1}^{n-1}\left(\tilde{\zeta}\left(\tilde{\Psi}_{A}^{+}\right)^{*} \tilde{\zeta}^{2} e^{-\lambda^{+}} c_{j k} T \tilde{\Psi}_{A}^{+} \varphi_{j}, \varphi_{k}\right)_{0}+\text { smoother terms } .
\end{aligned}
$$

where smoother terms are $O\left(\|\varphi\|_{-1}^{2}\right)$ or better (and the constant may depend on $\left.A\right)$. One fact quickly computed and used implicitly above is that $\sigma\left(\left(\tilde{\Psi}_{A}^{+}\right)^{*} T\right)=\sigma\left(T\left(\tilde{\Psi}_{A}^{+}\right)^{*}\right)=\xi_{2 n-1} \tilde{\psi}_{A}^{+}(\xi)$ (up to smooth terms) when applied to $\varphi$. Next, we will compute $\sigma\left(\left(\tilde{\Psi}_{A}^{+}\right)^{*} \tilde{\zeta}_{\nu}^{2} e^{-\lambda^{+}} c_{j k}\right) \cdot \sigma\left(\tilde{\Psi}_{A}^{+}\right) \equiv$ 
1 on $\mathcal{C}^{+}$, so $\sigma\left(\left(\tilde{\Psi}_{A}^{+}\right)^{*}\right) \equiv 1$ on $\mathcal{C}^{+}$as well, and it follows that $\sigma\left(\left(\tilde{\Psi}_{A}^{+}\right)^{*}\right)=\tilde{\psi}_{A}^{+}(\xi)$ up to terms supported in $\mathcal{C}^{0} \backslash \mathcal{C}^{+}$. Thus, up to errors on $\mathcal{C}^{0} \backslash \mathcal{C}^{+}$,

$$
\sigma\left(\left(\tilde{\Psi}_{A}^{+}\right)^{*} \tilde{\zeta}_{\nu}^{2} e^{-\lambda^{+}} c_{j k}\right)=\sum_{\beta \geq 0} \frac{1}{\beta !} \partial_{\xi}^{\beta} \tilde{\psi}_{A}^{+}(\xi) D_{x}^{\beta}\left(\tilde{\zeta}_{\nu}^{2} e^{-\lambda^{+}} c_{j k}\right)=\tilde{\psi}_{A}^{+}(\xi) \tilde{\zeta}_{\nu}^{2} e^{-\lambda^{+}} c_{j k}
$$

and on $\mathcal{C}^{+}$

$$
\begin{aligned}
\sigma\left(\left(\tilde{\Psi}_{A}^{+}\right)^{*} \tilde{\zeta}_{\nu}^{2} e^{-\lambda^{+}} c_{j k} T \Psi_{A}^{+}\right) & =\sum_{\alpha} \frac{1}{\alpha !} \partial_{\xi}^{\alpha} \sigma\left(\left(\tilde{\Psi}_{A}^{+}\right)^{*} \tilde{\zeta}_{\nu}^{2} e^{-\lambda^{+}} c_{j k}\right) D_{x}^{\alpha} \sigma\left(T \tilde{\Psi}_{A}^{+}\right) \\
& =\sum_{\alpha} \frac{1}{\alpha !} \partial_{\xi}^{\alpha}\left(\tilde{\psi}_{A}^{+}(\xi) \tilde{\zeta}_{\nu}^{2} e^{-\lambda^{+}} c_{j k}\right) D_{x}^{\alpha} \sigma\left(\xi_{2 n-1} \tilde{\psi}_{A}^{+}(\xi)\right)=\tilde{\zeta}_{\nu}^{2} e^{-\lambda^{+}} c_{j k} \xi_{2 n-1} .
\end{aligned}
$$

By construction, $\xi_{2 n-1} \geq A$ on $\mathcal{C}^{+}$and $\left(\tilde{\zeta}_{\nu} e^{-\lambda^{+}} c_{j k}\right)$ is positive semi-definite (and hence the sum of any $q$ eigenvalues is nonnegative), so we can apply Corollary 4.5 with $T$ as $R$ and $\left(e^{-\lambda^{+}} c_{j k}\right)$ as $\left(h_{j k}\right)$ to conclude that there exists a constant $C$ independent of $A$ so that

$$
\begin{aligned}
\sum_{J \in \mathcal{I}_{q}^{\prime}} & \left(c_{j j} T \varphi_{J}, \varphi_{J}\right)_{\lambda^{+}}-\sum_{J, J^{\prime} \in \mathcal{I}_{q}^{\prime}} \sum_{\substack{1 \leq j, k \leq m \\
j \neq k}} \epsilon_{j J^{\prime}}^{k J}\left(c_{j k} T \varphi_{J}, \varphi_{J^{\prime}}\right)_{\lambda^{+}} \\
\geq & A\left(\sum_{J \in \mathcal{I}_{q}^{\prime}}\left(\tilde{\zeta}^{2} e^{-\lambda^{+}} c_{j j} \varphi_{J}, \varphi_{J}\right)_{0}-\sum_{\substack { J, J^{\prime} \in \mathcal{I}_{q}^{\prime} \\
\begin{subarray}{c}{1 \leq j, k \leq m \\
j \neq k{ J , J ^ { \prime } \in \mathcal { I } _ { q } ^ { \prime } \\
\begin{subarray} { c } { 1 \leq j , k \leq m \\
j \neq k } }\end{subarray}} \epsilon_{j J^{\prime}}^{k J}\left(\tilde{\zeta}^{2} e^{-\lambda^{+}} c_{j k} \varphi_{J}, \varphi_{J^{\prime}}\right)_{0}\right) \\
& -C\|\varphi\|_{\lambda^{+}}^{2}+O\left(\|\varphi\|_{-1}^{2}\right)+O_{A}\left(\left\|\tilde{\zeta}_{\nu} \tilde{\Psi}_{A}^{0} \varphi\right\|_{0}^{2}\right. \\
= & A \sum_{J \in \mathcal{I}_{q}^{\prime}}\left(c_{j j} \varphi_{J}, \varphi_{J}\right)_{\lambda^{+}}-\sum_{J, J^{\prime} \in \mathcal{I}_{q}^{\prime}} \sum_{\substack{1 \leq j, k \leq m \\
j \neq k}} \epsilon_{j J^{\prime}}^{k J}\left(c_{j k} \varphi_{J}, \varphi_{J^{\prime}}\right)_{\lambda^{+}}+O\left(\|\varphi\|_{0}^{2}\right)+O_{A}\left(\left\|\tilde{\zeta} \tilde{\Psi}_{A}^{0} \varphi\right\|_{0}^{2}\right) .
\end{aligned}
$$

By the same argument, we have the following:

Lemma 4.7. Let $\varphi$ be a $(0, q)$-form supported on $U$ so that up to a smooth term, $\hat{\varphi}$ is supported in $\mathcal{C}^{-}$, then

$$
\begin{aligned}
& \sum_{J \in \mathcal{I}_{q}^{\prime}}\left(c_{j j}(-T) \varphi_{J}, \varphi_{J}\right)_{\lambda^{-}}+\sum_{J, J^{\prime} \in \mathcal{I}_{q}^{\prime}} \sum_{\substack{1 \leq j, k \leq m \\
j \neq k}} \epsilon_{j J^{\prime}}^{k J}\left(c_{j k}(-T) \varphi_{J}, \varphi_{J^{\prime}}\right)_{\lambda^{-}} \\
\geq & A\left(\sum_{J \in \mathcal{I}_{q}^{\prime}}\left(c_{j j} \varphi_{J}, \varphi_{J}\right)_{\lambda^{-}}+\sum_{J, J^{\prime} \in \mathcal{I}_{q}^{\prime}} \sum_{\substack{1 \leq j, k \leq m \\
j \neq k}} \epsilon_{j J^{\prime}}^{k J}\left(c_{j k} \varphi_{J}, \varphi_{J^{\prime}}\right)_{\lambda^{-}}\right)+O\left(\|\varphi\|_{\lambda^{-}}^{2}\right)+O_{A}\left(\left\|\tilde{\zeta}_{\nu} \tilde{\Psi}_{A}^{0} \varphi\right\|_{0}^{2}\right) .
\end{aligned}
$$

We now review the two local results from [Nic06] that are crucial in proving the basic estimate Proposition 4.1, Let $\left(s_{j k}^{+}\right)_{j, k=1}^{n-1}$ be the matrix defined by

$$
s_{j k}^{+}=\frac{1}{2}\left(\bar{L}_{k} L_{j}\left(\lambda^{+}\right)+L_{j} \bar{L}_{k}\left(\lambda^{+}\right)+\sum_{\ell=1}^{n-1}\left(d_{j k}^{\ell} L_{\ell}\left(\lambda^{+}\right)+\bar{d}_{k j}^{\ell} \bar{L}_{\ell}\left(\lambda^{+}\right)\right)\right)+A_{0} c_{j k} .
$$


Proposition 4.8. Let $\varphi \in \operatorname{Dom}\left(\bar{\partial}_{b}\right) \cap \operatorname{Dom}\left(\bar{\partial}_{b}^{*}\right)$ be a $(0, q)$-form supported in $U$. Assume that $\lambda^{+}$is a strictly CR-plurisubharmonic function on $(0, q)$-forms with CR-plurisubharmonicity constant $A_{\lambda^{+}}$. Then there exists a constant $C$ that is independent of $A_{\lambda^{+}}$so that

$$
Q_{b,+}\left(\tilde{\zeta} \Psi_{A}^{+} \varphi, \tilde{\zeta} \Psi_{A}^{+} \varphi\right)+C\left\|\tilde{\zeta} \Psi_{A}^{+} \varphi\right\|_{\lambda^{+}}^{2}+O_{\lambda^{+}}\left(\left\|\tilde{\zeta} \tilde{\Psi}_{A}^{0} \varphi\right\|_{0}^{2}\right) \geq A_{\lambda^{+}}\left\|\tilde{\zeta} \Psi_{A}^{+} \varphi\right\|_{\lambda^{+}}^{2} .
$$

Proof. Since $\varphi \in \operatorname{Dom}\left(\bar{\partial}_{b}\right) \cap \operatorname{Dom}\left(\bar{\partial}_{b}^{*}\right)$, it follows that $\tilde{\zeta} \Psi_{A}^{+} \varphi \in \operatorname{Dom}\left(\bar{\partial}_{b}\right) \cap \operatorname{Dom}\left(\bar{\partial}_{b}^{*}\right)$. Moreover, $\operatorname{supp}\left(\tilde{\zeta} \Psi_{A}^{+} \varphi\right) \subset U^{\prime}$. By Lemma 4.2,

$$
\begin{aligned}
& Q_{b,+}\left(\tilde{\zeta} \Psi_{A}^{+} \varphi, \tilde{\zeta} \Psi_{A}^{+} \varphi\right) \geq\left(1-\epsilon^{\prime}\right) \sum_{J \in \mathcal{I}_{q}^{\prime}} \sum_{j=1}^{n-1}\left\|\bar{L}_{j} \tilde{\zeta} \Psi_{A}^{+} \varphi_{J}\right\|_{\lambda^{+}}^{2} \\
& +\operatorname{Re}\left\{\sum_{J \in \mathcal{I}_{q}^{\prime}} \sum_{j \in J}\left(c_{j j} T \tilde{\zeta} \Psi_{A}^{+} \varphi_{J}, \tilde{\zeta} \Psi_{A}^{+} \varphi_{J}\right)_{\lambda^{+}}-\sum_{J, J^{\prime} \in \mathcal{I}_{q}^{\mathcal{I}_{q}}} \sum_{\substack{1 \leq j, k \leq n-1 \\
j \neq k}} \epsilon_{j J^{\prime}}^{k J}\left(c_{j k} T \tilde{\zeta} \Psi_{A}^{+} \varphi_{J}, \tilde{\zeta} \Psi_{A}^{+} \varphi_{J^{\prime}}\right)_{\lambda^{+}}\right\} \\
& +\frac{1}{2} \sum_{J \in \mathcal{I}_{q}^{\prime}} \sum_{j \in J}\left[\left(\left(\bar{L}_{j} L_{j}\left(\lambda^{+}\right)+L_{j} \bar{L}_{j}\left(\lambda^{+}\right)\right) \tilde{\zeta} \Psi_{A}^{+} \varphi_{J}, \tilde{\zeta} \Psi_{A}^{+} \varphi_{J}\right)_{\lambda^{+}}\right) \\
& \left.+\sum_{\ell=1}^{n-1}\left(\left(d_{j j}^{\ell} L_{\ell}\left(\lambda^{+}\right)+\bar{d}_{j j}^{\ell} \bar{L}_{\ell}\left(\lambda^{+}\right)\right) \tilde{\zeta} \Psi_{A}^{+} \varphi_{J}, \tilde{\zeta} \Psi_{A}^{+} \varphi_{J}\right)_{\lambda^{+}}\right] \\
& \sum_{J, J^{\prime} \in \mathcal{I}_{q}^{\prime}} \sum_{\substack{1 \leq j, k \leq n-1 \\
j \neq k}} \epsilon_{j J^{\prime}}^{k J}\left[\left(\left(\bar{L}_{k} L_{j}\left(\lambda^{+}\right)+L_{j} \bar{L}_{k}\left(\lambda^{+}\right)\right) \tilde{\zeta} \Psi_{A}^{+} \varphi_{J}, \tilde{\zeta} \Psi_{A}^{+} \varphi_{J^{\prime}}\right)_{\lambda^{+}}\right) \\
& \left.+\sum_{\ell=1}^{n-1}\left(\left(d_{j k}^{\ell} L_{\ell}\left(\lambda^{+}\right)+\bar{d}_{k j}^{\ell} \bar{L}_{\ell}\left(\lambda^{+}\right)\right) \tilde{\zeta} \Psi_{A}^{+} \varphi_{J}, \tilde{\zeta} \Psi_{A}^{+} \varphi_{J^{\prime}}\right)_{\lambda^{+}}\right]+O\left(\left\|\tilde{\zeta} \Psi_{A}^{+} \varphi\right\|_{0}^{2}\right) .
\end{aligned}
$$

To control the $T$ terms, we use Lemma 4.6 since supp $\tilde{\zeta} \subset U^{\prime}$, and the Fourier transform of $\tilde{\zeta} \Psi_{A}^{+} \varphi$ is supported in $\mathcal{C}^{+}$up to a smooth term. Indeed, with $A=A_{0}$ (and $A_{0}$ from the definition of $\left.\left(\mathrm{CR}-P_{q}\right)\right)$, we have

$$
\begin{aligned}
& \operatorname{Re}\left\{\sum_{J \in \mathcal{I}_{q}^{\prime}} \sum_{j \in J}\left(c_{j j} T \tilde{\zeta} \Psi_{A}^{+} \varphi_{J}, \tilde{\zeta} \Psi_{A}^{+} \varphi_{J}\right)_{\lambda^{+}}-\sum_{J, J^{\prime} \in \mathcal{I}_{q}^{\prime}} \sum_{\substack{1 \leq j, k \leq n-1 \\
j \neq k}} \epsilon_{j J^{\prime}}^{k J}\left(c_{j k} T \tilde{\zeta} \Psi_{A}^{+} \varphi_{J}, \tilde{\zeta} \Psi_{A}^{+} \varphi_{J^{\prime}}\right)_{\lambda^{+}}\right\}
\end{aligned}
$$

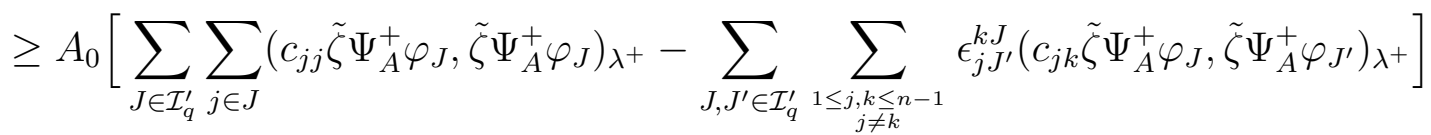

$$
\begin{aligned}
& +O\left(\left\|\tilde{\zeta} \Psi_{A}^{+} \varphi\right\|_{\lambda^{+}}^{2}\right)+O_{\lambda^{+}}\left(\left\|\tilde{\zeta} \tilde{\Psi}_{A}^{0} \varphi\right\|_{0}^{2}\right)
\end{aligned}
$$


Putting these estimates together, we have

$$
\begin{aligned}
& Q_{b,+}\left(\tilde{\zeta} \Psi_{A}^{+} \varphi, \tilde{\zeta} \Psi_{A}^{+} \varphi\right) \geq \\
& \sum_{J \in \mathcal{I}_{q}^{\prime}} \sum_{j \in J}\left(s_{j j}^{+} \tilde{\zeta} \Psi_{A}^{+} \varphi_{J}, \tilde{\zeta} \Psi_{A}^{+} \varphi_{J}\right)_{\lambda^{+}}-\sum_{J, J^{\prime} \in \mathcal{I}_{q}^{\prime}} \sum_{\substack{1 \leq j, k \leq n-1 \\
j \neq k}} \epsilon_{j J^{\prime}}^{k J}\left(s_{j k}^{+} \tilde{\zeta} \Psi_{A}^{+} \varphi_{J}, \tilde{\zeta} \Psi_{A}^{+} \varphi_{J^{\prime}}\right)_{\lambda^{+}} \\
& \left.+O\left(\left\|\tilde{\zeta} \Psi_{A}^{+} \varphi\right\|_{\lambda^{+}}^{2}\right)+O_{\lambda^{+}}\left(\left\|\tilde{\zeta} \tilde{\Psi}_{A}^{0} \varphi\right\|_{0}^{2}\right)\right)
\end{aligned}
$$

Recall that $\lambda^{+}$is strictly CR-plurisubharmonic on $(0, q)$-forms with CR-plurisubharmonicity constant $A_{\lambda^{+}}$. In local coordinates, if $L=\sum_{j=1}^{n-1} \xi_{j} L_{j}$, then

$$
\left\langle\frac{1}{2}\left(\partial_{b} \bar{\partial}_{b} \lambda^{+}-\bar{\partial}_{b} \partial_{b} \lambda^{+}\right)+A_{0} d \gamma, L \wedge \bar{L}\right\rangle=\sum_{j, k=1}^{n-1} s_{j k}^{+} \xi_{j} \bar{\xi}_{k}
$$

and $\left(s_{j k}^{+}\right)$is a Hermitian matrix. Therefore, by the multilinear algebra lemmas, Lemma A.1 and Lemma A.2.

$$
Q_{b,+}\left(\tilde{\zeta} \Psi_{A}^{+} \varphi, \tilde{\zeta} \Psi_{A}^{+} \varphi\right)+C\left\|\tilde{\zeta} \Psi_{A}^{+} \varphi\right\|_{\lambda^{+}}^{2}+O_{\lambda^{+}}\left(\left\|\tilde{\zeta} \tilde{\Psi}_{A}^{0} \varphi\right\|_{0}^{2}\right) \geq A_{\lambda^{+}}\left\|\tilde{\zeta} \Psi_{A}^{+} \varphi\right\|_{\lambda^{+}}^{2}
$$

where the constant $C$ is independent of $A_{\lambda^{+}}$.

Let

$$
s_{j k}^{-}=\frac{1}{2}\left(\bar{L}_{k} L_{j}\left(\lambda^{-}\right)+L_{j} \bar{L}_{k}\left(\lambda^{-}\right)+\sum_{\ell=1}^{n-1}\left(d_{j k}^{\ell} L_{\ell}\left(\lambda^{-}\right)+\bar{d}_{k j}^{\ell} \bar{L}_{\ell}\left(\lambda^{-}\right)\right)\right)+A_{0} c_{j k} .
$$

Proposition 4.9. Let $\varphi \in \operatorname{Dom}\left(\bar{\partial}_{b}\right) \cap \operatorname{Dom}\left(\bar{\partial}_{b}^{*}\right)$ be a $(0, q)$-form supported in $U$. Assume that $\lambda^{-}$is a strictly CR-plurisubharmonic function on $(0, n-1-q)$-forms with CRplurisubharmonicity constant $A_{\lambda^{-}}$Then there exists a constant $C$ that is independent of $A_{\lambda^{-}}$ so that

$$
Q_{b,-}\left(\tilde{\zeta} \Psi_{A}^{-} \varphi, \tilde{\zeta} \Psi_{A}^{-} \varphi\right)+C\left\|\tilde{\zeta} \Psi_{A}^{-} \varphi\right\|_{\lambda^{+}}^{2}+O_{\lambda^{-}}\left(\left\|\tilde{\zeta} \tilde{\Psi}_{A}^{0} \varphi\right\|_{0}^{2}\right) \geq A_{\lambda^{-}}\left\|\tilde{\zeta} \Psi_{A}^{-} \varphi\right\|_{\lambda^{-}}^{2}
$$


Proof. Similarly to the proof of Lemma 4.8, we can apply Lemma 4.3 to $\tilde{\zeta} \Psi_{A}^{-} \varphi$ which gives (for some $1 \gg \epsilon>0$ )

$$
\begin{aligned}
& Q_{b,-}\left(\tilde{\zeta} \Psi_{A}^{-} \varphi, \tilde{\zeta} \Psi_{A}^{-} \varphi\right) \geq\left(1-\epsilon^{\prime}\right) \sum_{J \in \mathcal{I}_{q}^{\prime}} \sum_{j=1}^{n-1}\left\|\bar{L}_{j}^{*,-} \tilde{\zeta} \Psi_{A}^{-} \varphi_{J}\right\|_{\lambda^{-}}^{2} \\
& +\operatorname{Re}\left\{\sum_{J \in \mathcal{I}_{q}^{\prime}} \sum_{j \in J}\left(c_{j j}(-T) \tilde{\zeta} \Psi_{A}^{-} \varphi_{J}, \tilde{\zeta} \Psi_{A}^{-} \varphi_{J}\right)_{\lambda^{-}}+\sum_{J, J^{\prime} \in \mathcal{I}_{q}^{\prime}} \sum_{\substack{1 \leq j, k \leq n-1 \\
j \neq k}} \epsilon_{j J^{\prime}}^{k J}\left(c_{j k}(-T) \tilde{\zeta} \Psi_{A}^{-} \varphi_{J}, \tilde{\zeta} \Psi_{A}^{-} \varphi_{J^{\prime}}\right)_{\lambda^{-}}\right\} \\
& +\frac{1}{2} \sum_{J \in \mathcal{I}_{q}^{\prime}} \sum_{j \in J}\left[\left(\left(\bar{L}_{j} L_{j}\left(\lambda^{+}\right)+L_{j} \bar{L}_{j}\left(\lambda^{+}\right)\right) \tilde{\zeta} \Psi_{A}^{-} \varphi_{J}, \tilde{\zeta} \Psi_{A}^{-} \varphi_{J}\right)_{\lambda^{+}}\right) \\
& \left.+\frac{1}{2} \sum_{J, J^{\prime} \in \mathcal{I}_{q}^{\prime}} \sum_{\substack{1 \leq j, k \leq n-1 \\
j \neq k}} \epsilon_{j J^{\prime}}^{k J}\left[\left(\left(\bar{L}_{k} L_{j}\left(\lambda^{-}\right)+L_{j}\left(\left(d_{j j}^{\ell} L_{\ell}\left(\lambda^{-}\right)+\bar{d}_{j j}^{\ell}\left(\lambda_{\ell}^{-}\right)\right) \tilde{\zeta} \Psi_{A}^{-} \varphi_{J}, \tilde{\zeta} \Psi_{A}^{-} \varphi_{J^{\prime}}\right)_{\lambda^{-}}\right)\right) \tilde{\zeta} \Psi_{A}^{-} \varphi_{J}, \tilde{\zeta} \Psi_{A}^{-} \varphi_{J}\right)_{\lambda^{-}}\right] \\
& \left.+\sum_{\ell=1}^{n-1}\left(\left(d_{j k}^{\ell} L_{\ell}\left(\lambda^{-}\right)+\bar{d}_{k j}^{\ell} \bar{L}_{\ell}\left(\lambda^{-}\right)\right) \tilde{\zeta} \Psi_{A}^{-} \varphi_{J}, \tilde{\zeta} \Psi_{A}^{-} \varphi_{J^{\prime}}\right)_{\lambda^{-}}\right]+O\left(\left\|\tilde{\zeta} \Psi_{A}^{-} \varphi\right\|_{0}^{2}\right) .
\end{aligned}
$$

To control the $T$ terms, we use Lemma 4.7 since supp $\tilde{\zeta} \subset U^{\prime}$, and the Fourier transform of $\tilde{\zeta} \Psi_{A}^{-} \varphi$ is supported in $\mathcal{C}^{-}$up to a smooth term. Indeed, with $A=A_{0}$ where $A_{0}$ is from the definition of CR-plurisubharmonicity on $(0, q)$-forms,

$$
\begin{array}{r}
\operatorname{Re}\left\{\sum_{J \in \mathcal{I}_{q}^{\prime}} \sum_{j \in J}\left(c_{j j}(-T) \tilde{\zeta} \Psi_{A}^{-} \varphi_{J}, \tilde{\zeta} \Psi_{A}^{-} \varphi_{J}\right)_{\lambda^{-}}+\sum_{J, J^{\prime} \in \mathcal{I}_{q}^{\prime}} \sum_{\substack{1 \leq j, k \leq n-1 \\
j \neq k}} \epsilon_{j J^{\prime}}^{k J}\left(c_{j k}(-T) \tilde{\zeta} \Psi_{A}^{-} \varphi_{J}, \tilde{\zeta} \Psi_{A}^{-} \varphi_{J^{\prime}}\right)_{\lambda^{-}}\right\} \\
\geq A_{0}\left[\sum_{J \in \mathcal{I}_{q}^{\prime}} \sum_{j \in J}\left(c_{j j} \tilde{\zeta} \Psi_{A}^{-} \varphi_{J}, \tilde{\zeta} \Psi_{A}^{-} \varphi_{J}\right)_{\lambda^{-}}+\sum_{\substack { J, J^{\prime} \in \mathcal{I}_{q}^{\prime} \\
\begin{subarray}{c}{1 \leq j, k \leq n-1 \\
j \neq k{ J , J ^ { \prime } \in \mathcal { I } _ { q } ^ { \prime } \\
\begin{subarray} { c } { 1 \leq j , k \leq n - 1 \\
j \neq k } }\end{subarray}} \epsilon_{j J^{\prime}}^{k J}\left(c_{j k} \tilde{\zeta} \Psi_{A}^{-} \varphi_{J}, \tilde{\zeta} \Psi_{A}^{+} \varphi_{J^{\prime}}\right)_{\lambda^{-}}\right] \\
+O\left(\left\|\tilde{\zeta} \Psi_{A}^{-} \varphi\right\|_{\lambda^{-}}^{2}\right)+O_{\lambda^{-}}\left(\left\|\tilde{\zeta} \tilde{\Psi}_{A}^{0} \varphi\right\|_{0}^{2}\right)
\end{array}
$$

Putting these estimates together, we have

$$
\begin{aligned}
& Q_{b,-}\left(\tilde{\zeta} \Psi_{A}^{-} \varphi, \tilde{\zeta} \Psi_{A}^{-} \varphi\right) \geq \\
& \sum_{J \in \mathcal{I}_{q}^{\prime}} \sum_{j \in J}\left(s_{j j}^{-} \tilde{\zeta} \Psi_{A}^{-} \varphi_{J}, \tilde{\zeta} \Psi_{A}^{-} \varphi_{J}\right)_{\lambda^{-}}+\sum_{\substack { J, J^{\prime} \in \mathcal{I}_{q}^{\prime} \\
\begin{subarray}{c}{1 \leq j, k \leq n-1 \\
j \neq k{ J , J ^ { \prime } \in \mathcal { I } _ { q } ^ { \prime } \\
\begin{subarray} { c } { 1 \leq j , k \leq n - 1 \\
j \neq k } }\end{subarray}} \epsilon_{j J^{\prime}}^{k J}\left(s_{j k}^{-} \tilde{\zeta} \Psi_{A}^{-} \varphi_{J}, \tilde{\zeta} \Psi_{A}^{-} \varphi_{J^{\prime}}\right)_{\lambda^{-}} \\
& \left.+O\left(\left\|\tilde{\zeta} \Psi_{A}^{-} \varphi\right\|_{\lambda^{-}}^{2}\right)+O_{\lambda^{-}}\left(\left\|\tilde{\zeta} \tilde{\Psi}_{A}^{0} \varphi\right\|_{0}^{2}\right)\right) .
\end{aligned}
$$


Recall that $\lambda^{-}$is strictly CR-plurisubharmonic on $(0, n-1-q)$-forms with CR-plurisubharmonicity constant $A_{\lambda^{-}}$. In local coordinates, if $L=\sum_{j=1}^{n-1} \xi_{j} L_{j}$, then

$$
\left\langle\frac{1}{2}\left(\partial_{b} \bar{\partial}_{b} \lambda^{-}-\bar{\partial}_{b} \partial_{b} \lambda^{-}\right)+A_{0} d \gamma, L \wedge \bar{L}\right\rangle=\sum_{j, k=1}^{n-1} s_{j k}^{-} \xi_{j} \bar{\xi}_{k}
$$

and $\left(s_{j k}^{-}\right)$is a Hermitian matrix. Therefore, by the multilinear algebra lemmas, Lemma A.1 and Lemma A.3.

$$
Q_{b,+}\left(\tilde{\zeta} \Psi_{A}^{+} \varphi, \tilde{\zeta} \Psi_{A}^{+} \varphi\right)+C\left\|\tilde{\zeta} \Psi_{A}^{+} \varphi\right\|_{\lambda^{+}}^{2}+O_{\lambda^{+}}\left(\left\|\tilde{\zeta} \tilde{\Psi}_{A}^{0} \varphi\right\|_{0}^{2}\right) \geq A_{\lambda^{+}}\left\|\tilde{\zeta} \Psi_{A}^{+} \varphi\right\|_{\lambda^{+}}^{2}
$$

where the constant $C$ is independent of $A_{\lambda^{+}}$.

We are finally ready to prove the basic estimate.

Proof. (Basic Estimate - Proposition 4.1). From (4), there exist constants $K, K_{ \pm}$so that if $A_{ \pm}=\min \left\{A_{\lambda^{-}}, A_{\lambda^{+}}\right\}$, then

$$
\begin{aligned}
K Q_{b, \pm}(\varphi, \varphi)+K_{ \pm} & \sum_{\nu}\left\|\tilde{\zeta}_{\nu} \tilde{\Psi}_{\nu, A}^{0} \zeta_{\nu} \varphi^{\nu}\right\|_{0}^{2}+K^{\prime}\|\varphi\|_{0}^{2}+O_{ \pm}\left(\|\varphi\|_{-1}^{2}\right) \\
& \geq \sum_{\nu}\left[Q_{b,+}\left(\tilde{\zeta}_{\nu} \Psi_{\nu, A}^{+} \zeta_{\nu} \varphi^{\nu}, \tilde{\zeta}_{\nu} \Psi_{\nu, A}^{+} \zeta_{\nu} \varphi^{\nu}\right)+Q_{b,-}\left(\tilde{\zeta}_{\nu} \Psi_{\nu, A}^{-} \zeta_{\nu} \varphi^{\nu}, \tilde{\zeta}_{\nu} \Psi_{\nu, A}^{-} \zeta_{\nu} \varphi^{\nu}\right)\right] .
\end{aligned}
$$

From Proposition 4.8 and Proposition 4.9 it follows that by increasing the size of $K, K_{ \pm}$, and $K^{\prime}$ (where $K^{\prime}$ does NOT depend on $A$ ) that

$$
K Q_{b, \pm}(\varphi, \varphi)+K_{ \pm} \sum_{\nu}\left\|\tilde{\zeta}_{\nu} \tilde{\Psi}_{\nu, A}^{0} \zeta_{\nu} \varphi^{\nu}\right\|_{0}^{2}+K^{\prime}\|\varphi\|_{0}^{2}+O_{ \pm}\left(\|\varphi\|_{-1}^{2}\right) \geq A_{ \pm}\|\varphi\|_{0}^{2}
$$

4.2. A Sobolev estimate in the "elliptic directions". For forms whose Fourier transforms are supported up to a smooth term in $\mathcal{C}^{0}$, we have better estimates. The following result is the $(0, q)$-form version of Lemma 4.18 in [Nic06].

Lemma 4.10. Let $\varphi$ be a $(0,1)$-form supported in $U_{\nu}$ for some $\nu$ such that up to a smooth term, $\hat{\varphi}$ is supported in $\tilde{\mathcal{C}}_{\nu}^{0}$. There exist positive constants $C>1$ and $C_{1}>0$ independent of A so that

$$
C Q_{b, \pm}\left(\varphi, E_{ \pm} \varphi\right)+C_{1}\|\varphi\|_{0}^{2} \geq\|\varphi\|_{1}^{2}
$$

The proof in [Nic06] also holds at level $(0, q)$.

We can use Lemma 4.10 to control terms of the form $\left\|\tilde{\zeta}_{\nu} \Psi_{\nu, A}^{0} \zeta_{\nu} \varphi^{\nu}\right\|_{0}^{2}$.

Proposition 4.11. For any $\epsilon>0$, there exists $C_{\epsilon, \pm}>0$ so that

$$
\left\|\tilde{\zeta}_{\nu} \Psi_{\nu, A}^{0} \zeta_{\nu} \varphi^{\nu}\right\|_{0}^{2} \leq \epsilon Q_{b, \pm}\left(\varphi^{\nu}, \varphi^{\nu}\right)+C_{ \pm}\left\|\varphi^{\nu}\right\|_{-1}^{2} .
$$


Proof. Observe that $\left\|\tilde{\zeta}_{\nu} \Psi_{\nu, A}^{0} \zeta_{\nu} \varphi^{\nu}\right\|_{0}^{2}=\left\|\Lambda^{-1} \tilde{\zeta}_{\nu} \Psi_{\nu, A}^{0} \zeta_{\nu} \varphi^{\nu}\right\|_{1}^{2}$. The $(0, q)$-form $\tilde{\zeta}_{\nu} \Psi_{\nu, A}^{0} \zeta_{\nu} \varphi^{\nu}$ is supported in $\mathcal{C}^{0}$, so Lemma 4.10 applies. Although the range of $\Lambda^{-1}$ is outside $U_{\nu}$, we can write $\Lambda^{-1} \tilde{\zeta}_{\nu}=\zeta_{\nu}^{\prime} \Lambda^{-1} \tilde{\zeta}_{\nu}+\left(1-\zeta_{\nu}^{\prime}\right) \Lambda^{-1} \tilde{\zeta}_{\nu}$ where $\zeta_{\nu}^{\prime}$ is a smooth bump function that is identically one on the support of $\tilde{\zeta}_{\nu}$. Then $\left(1-\zeta_{\nu}^{\prime}\right) \Lambda^{-1} \tilde{\zeta}_{\nu}$ is infinitely smoothing and hence can be absorbed in the $\|\varphi\|_{-1}^{2}$ term. Let $P=\zeta_{\nu}^{\prime} \Lambda^{-1}$ and $\psi=\tilde{\zeta}_{\nu} \Psi_{\nu, A}^{0} \zeta_{\nu} \varphi^{\nu}$. By Lemma 4.10 and the fact that $P$ is an order -1 pseudodifferential operator,

$$
\left\|\Lambda^{-1} \tilde{\zeta}_{\nu} \Psi_{\nu, A}^{0} \zeta_{\nu} \varphi^{\nu}\right\|_{1}^{2} \leq\|P \psi\|_{1}^{2}+C\left\|\varphi^{\nu}\right\|_{-1}^{2} \leq C_{1} Q_{b, \pm}(P \psi, P \psi)+C\left\|\varphi^{\nu}\right\|_{-1}^{2} .
$$

The adjoint of $P$ is $P^{*, \pm}=\zeta_{\nu}^{\prime} \Lambda^{-1}$. Consequently $P-P^{*, \pm}$ is an order -2 pseudodifferential operator, and we can apply Lemma 2.4.2 in FK72 to prove

$$
Q_{b, \pm}(P \psi, P \psi)=\operatorname{Re} Q_{b, \pm}\left(\psi, P^{*, \pm} P \psi\right)+C_{ \pm}\left\|\varphi^{\nu}\right\|_{-1}^{2} \leq \epsilon Q_{b, \pm}\left(\varphi^{\nu}, \varphi^{\nu}\right)+C_{\epsilon, \pm}\left\|\varphi^{\nu}\right\|_{-1}^{2} .
$$

The term $\epsilon Q_{b, \pm}(\varphi, \varphi)$ could be replaced by $\epsilon\left\|\square_{b, \pm} \varphi\right\|_{-1}^{2}$ if we had a need for it.

\section{Existence and Compactness Theorems for the Complex Green Operator}

In this section, we use the basic estimate to prove existence and compactness theorems for the complex Green operator. As always, $M$ is a compact, orientable, weakly pseudoconvex CR-manifold of dimension at least 5, endowed with strongly CR-plurisubharmonic functions $\lambda^{+}$and $\lambda^{-}$.

5.1. Closed range for $\square_{b, \pm}$. For $1 \leq q \leq n-2$, let

$$
\begin{aligned}
\mathcal{H}_{ \pm}^{q} & =\left\{\varphi \in \operatorname{Dom}\left(\bar{\partial}_{b}\right) \cap \operatorname{Dom}\left(\bar{\partial}_{b}^{*}\right): \bar{\partial}_{b} \varphi=0, \bar{\partial}_{b, \pm}^{*} \varphi=0\right\} \\
& =\left\{\varphi \in \operatorname{Dom}(\bar{\partial}) \cap \operatorname{Dom}\left(\bar{\partial}_{b}^{*}\right): Q_{b, \pm}(\varphi, \varphi)=0\right\}
\end{aligned}
$$

be the space of \pm -harmonic $(0, q)$-forms.

Lemma 5.1. For $A_{ \pm}$suitably large and $1 \leq q \leq n-2, \mathcal{H}_{ \pm}^{q}$ is finite dimensional and there exists $C$ that does not depend on $\lambda^{+}$and $\lambda^{-}$so that for all $(0, q)$-forms $\varphi \in \operatorname{Dom}\left(\bar{\partial}_{b}\right) \cap$ $\operatorname{Dom}\left(\bar{\partial}_{b}^{*}\right)$ so that $\varphi \perp \mathcal{H}_{ \pm}^{q}$ (with respect to $\langle\cdot, \cdot\rangle_{ \pm}$).

$$
\|\varphi\|_{ \pm}^{2} \leq C Q_{b, \pm}(\varphi, \varphi) .
$$

Proof. For $\varphi \in \mathcal{H}_{ \pm}$, we can use Proposition 4.1 with $A_{ \pm}$suitably large (to absorb terms) so that

$$
A_{ \pm}\|\varphi\|_{ \pm}^{2} \leq C_{ \pm}\left(\sum_{\nu}\left\|\tilde{\zeta}_{\nu} \Psi_{\nu, A}^{0} \zeta_{\mu} \varphi^{\nu}\right\|_{0}^{2}+\|\varphi\|_{-1}^{2}\right) .
$$

Also, by Proposition 4.11,

$$
\sum_{\nu}\left\|\tilde{\zeta}_{\nu} \Psi_{\nu, A}^{0} \zeta_{\mu} \varphi^{\nu}\right\|_{0}^{2} \leq C_{ \pm}\|\varphi\|_{-1}^{2}
$$

since $Q_{b, \pm}(\varphi, \varphi)=0$. The unit ball in $\mathcal{H}_{ \pm} \cap L^{2}(M)$ is compact, and hence finite dimensional. 
Assume that (7) fails. Then there exists $\varphi_{k} \perp \mathcal{H}_{ \pm}$with $\left\|\mid \varphi_{k}\right\|_{ \pm}=1$ so that

$$
\left\|\varphi_{k}\right\|_{ \pm}^{2} \geq k Q_{b, \pm}\left(\varphi_{k}, \varphi_{k}\right) .
$$

For $k$ suitably large, we can use Proposition 4.1 and the above argument to absorb $Q_{b, \pm}\left(\varphi_{k}, \varphi_{k}\right)$ by $A_{ \pm}\left|\left\|\varphi_{k} \mid\right\|_{ \pm}\right.$to get:

$$
\left\|\varphi_{k}\right\|_{ \pm}^{2} \leq C_{ \pm}\left\|\varphi_{k}\right\|_{-1}^{2}
$$

Since $H^{-1}(M)$ is compact in $L^{2}(K)$, there exists a subsequence $\varphi_{k_{j}}$ that converges in $L^{2}(M)$. Since $\left(Q_{b, \pm}(\cdot, \cdot)+\|\| \cdot \|_{ \pm}^{2}\right)^{1 / 2}$ is a norm that dominates the $L^{2}(M)$-norm, there is a further subsequence that converges in the $\left(Q_{b, \pm}(\cdot, \cdot)+\left|\|\cdot \mid\|_{ \pm}^{2}\right)^{1 / 2}\right.$ norm as well. The limit $\varphi$ satisfies $\|\varphi\|_{ \pm}=1$ and $\varphi \perp \mathcal{H}_{ \pm}$. But from the above inequality, $\varphi \in \mathcal{H}_{ \pm}$. This is a contradiction and (7) holds.

Let

$$
{ }^{\perp} \mathcal{H}_{ \pm}^{q}=\left\{\varphi \in L_{0, q}^{2}(M):\langle\varphi, \phi\rangle_{ \pm}=0, \text { for all } \phi \in \mathcal{H}_{ \pm}^{q}\right\}
$$

On ${ }^{\perp} \mathcal{H}_{ \pm}^{q}$, define

$$
\square_{b, \pm}=\bar{\partial}_{b} \bar{\partial}_{b, \pm}^{*}+\bar{\partial}_{b, \pm}^{*} \bar{\partial}_{b}
$$

Since $\bar{\partial}_{b, \pm}^{*}=E_{ \pm} \bar{\partial}_{b}^{*}+\left[\bar{\partial}_{b}^{*}, E_{ \pm}\right], \operatorname{Dom}\left(\bar{\partial}_{b, \pm}^{*}\right)=\operatorname{Dom}\left(\bar{\partial}_{b}^{*}\right)$. This causes

$\operatorname{Dom}\left(\square_{b, \pm}\right)=\left\{\varphi \in L_{0, q}^{2}(M): \varphi \in \operatorname{Dom}\left(\bar{\partial}_{b}\right) \cap \operatorname{Dom}\left(\bar{\partial}_{b}^{*}\right), \bar{\partial}_{b} \varphi \in \operatorname{Dom}\left(\bar{\partial}_{b}^{*}\right)\right.$, and $\left.\bar{\partial}_{b}^{*} \varphi \in \operatorname{Dom}\left(\bar{\partial}_{b}\right)\right\}$.

5.2. Proof of Theorem 1.1 when $s=0$. This subsection is devoted the proof of Theorem 1.1 when $s=0$, i.e., the $L^{2}$-case.

As a consequence of Lemma 5.1, we may apply Theorem 1.1.2 in Hör65] to conclude that $\bar{\partial}_{b}: L_{(0, q)}^{2}(M) \rightarrow L_{(0, q+1)}^{2}(M)$ and $\bar{\partial}_{b, \pm}^{*}: L_{(0, q)}^{2}(M) \rightarrow L_{(0, q-1)}^{2}(M)$ have closed range. However, by Theorem 1.1.1 in [Hör65], this also means that $\bar{\partial}_{b}: L_{(0, q-1)}^{2}(M) \rightarrow L_{(0, q)}^{2}(M)$ and $\bar{\partial}_{b, \pm}^{*}: L_{(0, q+1)}^{2}(M) \rightarrow L_{(0, q)}^{2}(M)$ have closed range (and satisfy the appropriate $L^{2}$ inequality with a constant that does NOT depend on $\lambda^{+}$or $\lambda^{-}$). Again by Lemma 5.1, Theorem 1.1.1 in [Hör65], and Lemma 3.5, $\bar{\partial}_{b}^{*}$ has closed range when acting on $L_{(0, q)}^{2}(M)$ or $L_{(0, q+1)}^{2}(M)$. Therefore, for a $(0, q)$-form $u \in \operatorname{Dom}\left(\bar{\partial}_{b}\right) \cap \operatorname{Dom}\left(\bar{\partial}_{b}^{*}\right)$, we have the estimates

$$
\|u\|_{0}^{2} \leq C\left(\left\|\bar{\partial}_{b} u\right\|_{0}^{2}+\left\|\bar{\partial}_{b}^{*} u\right\|_{0}^{2}+\left\|H_{q} u\right\|_{0}^{2}\right)
$$

and

$$
\|u\|_{0}^{2} \leq C\left(Q_{b, \pm}(u, u)+\left\|H_{ \pm, q} u\right\|_{0}^{2}\right)
$$

where $H_{q}$ is the projection of $u$ onto $\mathcal{H}^{q}$ and $H_{ \pm, q}$ is the projection of $u$ onto $\mathcal{H}_{ \pm}^{q}$. This implies the existence of $G_{q}$ and $G_{q, \pm}$ as bounded operators on $L_{(0, q)}^{2}(M)$ that invert $\square_{b}$ on $\mathcal{H}^{q}$ and $\square_{b, \pm}$ on $\mathcal{H}_{ \pm}^{q}$, respectively (see for example [Sha85a], Lemma 3.2 and its proof). Moreover, the solvability of $\bar{\partial}_{b}$ in $L_{(0, q)}^{2}(M)$ and weighted $L_{(0, q)}^{2}(M)$ forces

$$
\operatorname{ker}\left(\bar{\partial}_{b}\right)=\underbrace{\operatorname{Range}\left(\bar{\partial}_{b}\right) \oplus \mathcal{H}_{ \pm}^{q}}_{\oplus \text { with respect to }\langle\cdot, \cdot\rangle_{ \pm}}=\underbrace{\operatorname{Range}\left(\bar{\partial}_{b}\right) \oplus \mathcal{H}^{q}}_{\oplus \text { with respect to }(\cdot, \cdot)_{0}} .
$$

Consequently, $\mathcal{H}^{q}$ is finite dimensional. 
We now prove that $G_{q}$ is compact. First observe, we have the following identity:

$$
\begin{aligned}
G_{q+1} \bar{\partial}_{b} u=G_{q+1} \bar{\partial}_{b}\left(\bar{\partial}_{b} \bar{\partial}_{b}^{*}+\bar{\partial}_{b}^{*} \bar{\partial}_{b}\right) G_{q} u & =G_{q+1} \bar{\partial}_{b} \bar{\partial}_{b}^{*} \bar{\partial}_{b} G_{q} u \\
& =G_{q+1}\left(\bar{\partial}_{b} \bar{\partial}_{b}^{*}+\bar{\partial}_{b}^{*} \bar{\partial}_{b}\right) \bar{\partial}_{b} G_{q} u=\bar{\partial}_{b} G_{q} u .
\end{aligned}
$$

Thus,

$$
\bar{\partial}_{b} G_{q}=\left(\bar{\partial}_{b}^{*} G_{q+1}\right)^{*} .
$$

To prove compactness of $G_{q}$, it suffices to show compactness on ${ }^{\perp} \mathcal{H}^{q}$ (since $G_{q}$ is zero on $\mathcal{H}^{q}$ ). When $u \in{ }^{\perp} \mathcal{H}^{q}$, equation (8) implies (since $G_{q} u \in{ }^{\perp} \mathcal{H}^{q}$ )

$$
\left\|G_{q} u\right\|_{0}^{2} \lesssim\left\|\bar{\partial}_{b} G_{q} u\right\|_{0}^{2}+\left\|\bar{\partial}_{b}^{*} G_{q} u\right\|_{0}^{2}=\left\|\left(\bar{\partial}_{b}^{*} G_{q+1}\right)^{*} u\right\|_{0}^{2}+\left\|\bar{\partial}_{b}^{*} G_{q} u\right\|_{0}^{2}
$$

Therefore, we only need to show that both $\bar{\partial}_{b}^{*} G_{q}$ and $\bar{\partial}_{b}^{*} G_{q+1}$ are compact. Our main tool will be a strengthening of (9). We claim that

$$
\|u\|_{0}^{2} \leq \frac{C}{A_{ \pm}}\left(\left\|\bar{\partial}_{b} u\right\|_{ \pm}^{2}+\left\|\bar{\partial}_{b, \pm}^{*} u \mid\right\|_{ \pm}^{2}\right)+C_{ \pm}\|u\|_{-1}^{2} .
$$

To prove (11), we already know the estimate if $u \in \mathcal{H}_{ \pm}^{q}$, so we can assume that $u \in{ }^{\perp} \mathcal{H}_{ \pm}^{q}$. we use Proposition 4.1 to see that

$$
A_{ \pm} \mid\|u\|_{ \pm} \leq K Q_{b, \pm}(u, u)+K_{ \pm}\left(\sum_{\nu}\left\|\tilde{\zeta}_{\nu} \Psi_{\nu, A}^{0} \zeta_{\nu} u^{\nu}\right\|_{0}^{2}+\|u\|_{-1}^{2}\right)
$$

Thus, to prove (11), we have to show that $K_{ \pm} \sum_{\nu}\left\|\tilde{\zeta}_{\nu} \Psi_{\nu, A}^{0} \zeta_{\nu} u^{\nu}\right\|_{0}^{2}$ is well-controlled. Using Proposition 4.11, we have (with $\epsilon=1 / K_{ \pm}$),

$$
K_{ \pm} \sum_{\nu}\left\|\tilde{\zeta}_{\nu} \Psi_{\nu, A}^{0} \zeta_{\nu} u^{\nu}\right\|_{0}^{2} \leq Q_{b, \pm}(u, u)+K_{ \pm}^{\prime}\|u\|_{-1}^{2}
$$

and (11) is proved.

When $\alpha \in$ Range $\left(\bar{\partial}_{b}\right) \subset L_{0, q+1}^{2}(M), \bar{\partial}_{b}^{*} G_{q+1} \alpha$ gives the norm minimizing solution to $\bar{\partial}_{b} v=$ $\alpha, \alpha \in \operatorname{Range}\left(\bar{\partial}_{b}\right) \subset L_{0, q+1}^{2}(M)$, while $\bar{\partial}_{b, \pm}^{*} G_{ \pm, q+1} \alpha$ gives a different solution (the one that minimizes the $\mid\|\cdot\| \|_{ \pm}$-norm). For such $\alpha$, (11) therefore implies

$$
\begin{aligned}
\left\|\bar{\partial}_{b}^{*} G_{q+1} \alpha\right\|_{0}^{2} \leq & \left\|\bar{\partial}_{b, \pm}^{*} G_{ \pm, q+1} \alpha\right\|_{0}^{2} \leq C \mid\left\|\bar{\partial}_{b, \pm}^{*} G_{ \pm, q+1} \alpha\right\|_{ \pm}^{2} \\
& \leq \frac{C}{A_{ \pm}}\|\alpha\|_{ \pm}+C_{ \pm}\left\|\bar{\partial}_{b, \pm}^{*} G_{ \pm, q+1} \alpha\right\|_{-1}^{2} \leq \frac{C}{A_{ \pm}}\|\alpha\|\left\|_{ \pm}+C_{ \pm}\right\| \bar{\partial}_{b, \pm}^{*} G_{ \pm, q+1} \alpha \|_{-1}^{2}
\end{aligned}
$$

Applying Lemma 5.1 to $\bar{\partial}_{b, \pm}^{*} G_{ \pm, q+1}$ shows that $\bar{\partial}_{b, \pm}^{*} G_{ \pm, q+1}: L_{0, q+1}^{2}(M) \rightarrow L_{0, q+1}^{2}(M)$ is a bounded operator with $C$ is independent of $A_{ \pm}$. Therefore, $L_{0, q+1}^{2}(M)$ embeds compactly in $W_{0, q+1}^{-1}(M)$. Moreover, $A_{ \pm}$can be made arbitrarily large since $M$ satisfies $\left(P_{q}\right)$ and $\left(P_{n-1-q}\right)$. Equation (12) proves that $\bar{\partial}_{b, \pm}^{*} G_{ \pm, q+1}: L_{0, q+1}^{2}(M) \rightarrow L_{0, q}^{2}(M)$ continuously, so the map $\bar{\partial}_{b, \pm}^{*} G_{ \pm, q+1}: L_{0, q+1}^{2}(M) \rightarrow W_{0, q}^{-1}(M)$ is compact, and it follows that $\bar{\partial}_{b}^{*} G_{q+1}$ is compact on Range $\left(\bar{\partial}_{b}\right)$ by ['A02, Proposition V.2.3. On the orthogonal complement of Range $\left(\bar{\partial}_{b}\right)$, 
$\bar{\partial}_{b}^{*} G_{q+1}=0$, so $\bar{\partial}_{b}^{*} G_{q+1}: L_{0, q+1}^{2}(M) \rightarrow L_{0, q+1}^{2}(M)$ is compact. To estimate $\bar{\partial}_{b}^{*} G_{q} \alpha$, we cannot invoke (11) directly because $\bar{\partial}_{b}^{*} G_{q} \alpha$ is a $(q-1)$-form. Instead, for $\alpha \in \operatorname{Range}\left(\bar{\partial}_{b}\right) \subset L_{0, q}^{2}(M)$,

$$
\begin{aligned}
& \left\|\bar{\partial}_{b, \pm}^{*} G_{ \pm, q} \alpha\right\|_{ \pm}^{2}=\left\langle\bar{\partial}_{b} \bar{\partial}_{b, \pm}^{*} G_{ \pm, q} \alpha, G_{ \pm, q} \alpha\right\rangle_{ \pm}=\left\langle\alpha, G_{ \pm, q} \alpha\right\rangle_{ \pm} \\
& \leq \frac{2 C}{A_{ \pm}}\|\alpha \mid\|_{ \pm}^{2}+\frac{A_{ \pm}}{2 C}\left\|G_{ \pm, q} \alpha\right\|_{ \pm}^{2} \leq \frac{2 C}{A_{ \pm}}\|\alpha\|_{ \pm}^{2}+\frac{1}{2}\left\|\bar{\partial}_{b, \pm}^{*} G_{ \pm, q} \alpha\right\|\left\|_{ \pm}^{2}+C_{ \pm}\right\| G_{ \pm, q} \alpha \|_{-1}^{2} .
\end{aligned}
$$

Here we have used that $\bar{\partial}_{b} \alpha=0$ and that $\alpha \in{ }^{\perp} \mathcal{H}_{ \pm}^{q}$ (since $\alpha \in$ Range $\bar{\partial}_{b}$ ) in the second inequality. Also, the first inequality shows that the $\left\|\bar{\partial}_{b, \pm}^{*} G_{ \pm, q} \alpha\right\|_{ \pm}^{2}<\infty$ and thus the term in the final inequality can be absorbed. Thus we can can prove $\bar{\partial}_{b}^{*} G_{q} L_{0, q}^{2}(M) \rightarrow L_{0, q-1}^{2}(M)$ is a compact operator by repeating the argument that follows (12) with $G_{ \pm, q}$ replacing $\bar{\partial}_{b, \pm}^{*} G_{ \pm, q+1}$.

5.3. End proof of Theorem 1.1 - the $s>0$ case. Fix $s>0$. Recall that compactness $G_{q}$ in $L_{0, q}^{2}(M)$ is equivalent to the following compactness estimate: for every $\epsilon>0$, there exists $C_{\epsilon}>0$ so that for every $u \in \operatorname{Dom}\left(\bar{\partial}_{b}\right) \cap \operatorname{Dom}\left(\bar{\partial}_{b}^{*}\right)$,

$$
\|u\|_{0}^{2} \leq \epsilon\left(\left\|\bar{\partial}_{b} u\right\|_{0}^{2}+\left\|\bar{\partial}_{b}^{*} u\right\|_{0}^{2}\right)+C_{\epsilon}\|u\|_{-1}^{2} .
$$

We claim that this estimate also holds a priori in $H^{s}, s>0$. Indeed, using the fact that the commutators $\left[\bar{\partial}_{b}, \Lambda^{s}\right]$ and $\left[\bar{\partial}_{b}^{*}, \Lambda^{s}\right]$ are pseudodifferential operators of order $s$ (independent of $\epsilon)$, we have

$$
\begin{aligned}
\|u\|_{s}^{2} & =\left\|\Lambda^{s} u\right\|_{0}^{2} \leq \epsilon\left(\left\|\bar{\partial}_{b} \Lambda^{s} u\right\|_{0}^{2}+\left\|\bar{\partial}_{b}^{*} \Lambda^{s} u\right\|_{0}^{2}\right)+C_{\epsilon}\left\|\Lambda^{s} u\right\|_{-1}^{2} \\
& \leq \epsilon\left(\left\|\Lambda^{s} \bar{\partial}_{b} u\right\|_{0}^{2}+\left\|\Lambda^{s} \bar{\partial}_{b}^{*} u\right\|_{0}^{2}\right)+\epsilon\left(\left\|\left[\bar{\partial}_{b}, \Lambda^{s}\right] u\right\|_{0}^{2}+\left\|\left[\bar{\partial}_{b}^{*}, \Lambda^{s}\right] u\right\|_{0}^{2}\right) C_{\epsilon}\|u\|_{s-1}^{2} \\
& \leq \epsilon\left(\left\|\bar{\partial}_{b} u\right\|_{s}^{2}+\left\|\bar{\partial}_{b}^{*} u\right\|_{s}^{2}\right)+C \epsilon\|u\|_{s}^{2}+C_{\epsilon}\|u\|_{s-1}^{2} .
\end{aligned}
$$

When $\epsilon<1 / 2 C$, the $C \epsilon\|u\|_{s}^{2}$ can be absorbed into the left-hand side of the equation. Thus, we have the estimate that for every $\epsilon>0$, there exists $C_{\epsilon}>0$ so that for every $u \in H_{0, q}^{s}(M)$ with $\bar{\partial}_{b} u \in H_{0, q+1}^{s}(M)$ and $\bar{\partial}_{b}^{*} u \in H_{0, q-1}^{s}(M)$,

$$
\|u\|_{s}^{2} \leq \epsilon\left(\left\|\bar{\partial}_{b} u\right\|_{s}^{2}+\left\|\bar{\partial}_{b}^{*} u\right\|_{s}^{2}\right)+C_{\epsilon}\|u\|_{s-1}^{2} .
$$

Unlike in $L^{2}$-case, this estimate does not imply that $G_{q}$ is compact in $H^{s}$. The difficulty rests in the fact that while $u$ may be in $H_{0, q}^{s}(M)$, we can only say that $G_{q} u \in L_{0, q}^{2}(M)$. We need to work with the family of regularized operators $G_{\delta, q}, 0<\delta \leq 1$, arising from the following regularization. Let $Q_{b, 0}^{\delta}(\cdot, \cdot)$ be the quadratic form on $H_{0, q}^{1}(M)$ defined by

$$
Q_{b, 0}^{\delta}(u, v)=Q_{b, 0}(u, v)+\delta Q_{L}(u, v)
$$

where $Q_{L}$ is the hermitian inner product associated to the de Rham exterior derivative $d$, i.e., $Q_{L}(u, v)=(d u, d v)_{0}+\left(d^{*} u, d^{*} v\right)_{0}$. The inner product $Q_{L}$ has form domain $H_{0, q}^{1}(M)$. Consequently, $Q_{b, 0}^{\delta}$ gives rise a unique, self-adjoint, elliptic operator $\square_{b, \delta}$ with inverse $G_{q, \delta}$. Equivalently, for $u \in L_{0, q}^{2}(M)$ and $v \in H_{0, q}^{1}(M),(u, v)_{0}=Q_{b, 0}^{\delta}\left(G_{q, \delta} u, v\right)$. By elliptic regularity, we know that if $u \in H_{0, q}^{s}(M)$, then $G_{q, \delta} u \in H_{0, q}^{s+2}(M)$. We claim that for any $\epsilon>0$, there exists $C_{\epsilon}$ so that for any $u \in H_{0, q}^{s}(M)$,

$$
\left\|G_{q, \delta} u\right\|_{s}^{2} \leq \epsilon\|u\|_{s}^{2}+C_{\epsilon}\|u\|_{s-1}^{2},
$$


where the inequalities are uniform in $0<\delta \leq 1$. Estimates of the form (15) are well known to be equivalent to the compactness of $G_{q, \delta}$ on $H_{0, q}^{s}(M)$, (see, for example, [D'A02], Proposition V.2.3).

By the a priori estimate (14),

$$
\left\|G_{q, \delta} u\right\|_{s}^{2} \leq \epsilon\left(\left\|\bar{\partial}_{b} G_{q, \delta} u\right\|_{s}^{2}+\left\|\bar{\partial}_{b}^{*} G_{q, \delta} u\right\|_{s}^{2}\right)+C_{\epsilon}\|u\|_{s-1}^{2} .
$$

The $\bar{\partial}_{b}$ and $\bar{\partial}_{b}^{*}$ terms can be estimated as follows:

$$
\begin{aligned}
\left\|\bar{\partial}_{b} G_{q, \delta} u\right\|_{s}^{2}+\left\|\bar{\partial}_{b}^{*} G_{q, \delta} u\right\|_{s}^{2} & \leq Q_{b, 0}\left(\Lambda^{s} G_{q, \delta} u, \Lambda^{s} G_{q, \delta} u\right)+C\left\|G_{q, \delta} u\right\|_{s}^{2} \\
& \leq Q_{b, 0}^{\delta}\left(\Lambda^{s} G_{q, \delta} u, \Lambda^{s} G_{q, \delta} u\right)+C\left\|G_{q, \delta} u\right\|_{s}^{2} \\
& \leq\left|\left(\Lambda^{s} u, \Lambda^{s} G_{q, \delta} u\right)_{0}\right|+C\|u\|_{s}^{2},
\end{aligned}
$$

where we have used the estimate $Q_{b, 0}^{\delta}\left(\Lambda^{s} G_{q, \delta} u, \Lambda^{s} G_{q, \delta} u\right) \leq\left|\left(\Lambda^{s} u, \Lambda^{s} G_{q, \delta} u\right)_{0}\right|+C\left\|G_{q, \delta} u\right\|_{s}^{2}$, which follows from [KN65], Lemma 3.1. Thus, we have

$$
\left\|G_{q, \delta} u\right\|_{s}^{2} \leq \epsilon\left(\left\|G_{q, \delta} u\right\|_{s}^{2}+\|u\|_{s}^{2}\right)+C_{\epsilon}\|u\|_{s-1}^{2},
$$

By absorbing terms (and choosing $\epsilon<1 / 2$ ), we have proven (15) with the constant $C_{\epsilon}$ independent of $\delta, 0<\delta \leq 1$.

We want to let $\delta \rightarrow 0$. If $u \in H_{0, q}^{s}(M)$, then $\left\{G_{q, \delta} u: 0<\delta \leq 1\right\}$ is bounded in $H_{0, q}^{s}(M)$. Thus, there exists a sequence $\delta_{k} \rightarrow 0$ and $\tilde{u} \in H_{0, q}^{s}(M)$ so that $G_{q, \delta_{n}} u \rightarrow \tilde{u}$ weakly in $H_{0, q}^{s}(M)$. Consequently, if $v \in H_{0, q}^{1}(M)$, then

$$
\lim _{n \rightarrow \infty} Q_{b, 0}^{\delta_{n}}\left(G_{q, \delta_{n}} u, v\right)=Q_{b, 0}(\tilde{u}, v) .
$$

However,

$$
Q_{b, 0}^{\delta_{n}}\left(G_{q, \delta_{n}} u, v\right)=(u, v)=Q_{b, 0}\left(G_{q} u, v\right)
$$

so $G_{q} u=\tilde{u}$ and 15 is satisfied with $\delta=0$. Thus, $G_{q}$ is a compact operator on $H_{0, q}^{s}(\Omega)$. and Theorem 1.1 is proved.

\section{Appendix A. Multilinear Algebra}

Some crucial multilinear algebra is contained in the following lemma from Straube [Str].

Lemma A.1. Let $\left(\lambda_{j k}\right)_{j, k=1}^{m}(z)$ be an $m \times m$ matrix-valued function and $1 \leq q \leq m$. The following are equivalent:

(1) $\sum_{K \in \mathcal{I}_{q-1}} \sum_{j, k=1}^{m} \lambda_{j k}(z) u_{j K} \overline{u_{k K}} \geq A|u|^{2} \quad \forall u \in \Lambda_{z}^{(0, q)}$.

(2) The sum of any $q$ eigenvalues of $\left(\lambda_{j k}(z)\right)_{j, k}$ is at least $A$.

(3) For any orthonormal $\underline{t}^{\ell} \in \mathbb{C}^{m}, 1 \leq j \leq q$,

$$
\sum_{\ell=1}^{q} \lambda_{j k}(z)\left(\underline{t}^{\ell}\right)_{j} \overline{\left(\underline{t}^{\ell}\right)_{k}} \geq A
$$


These are Lemma 6.3 and Lemma 6.4 in Nic06].

Lemma A.2. Let $\left(b_{j k}\right)$ be a Hermitian matrix and let $1 \leq q \leq n-2$. Then then $\left(\begin{array}{c}n-1 \\ q\end{array}\right)$ by $\left(\begin{array}{c}n-1 \\ q\end{array}\right)$ matrix $\left(B_{J J^{\prime}}^{q}\right)$ given by

$$
\begin{aligned}
& B_{J J}^{q}=\sum_{j \in J} b_{j j} \\
& B_{J J^{\prime}}^{q}=-\sum_{\substack{1 \leq j, k \leq n-1 \\
j \neq k}} \epsilon_{j J^{\prime}}^{k J} b_{j k} \quad \text { if } J \neq J^{\prime},
\end{aligned}
$$

where $J$ and $J^{\prime}$ are multiindices, $|J|=\left|J^{\prime}\right|=q$ is also Hermitian. Moreover, the eigenvalues of $\left(B_{J J^{\prime}}^{q}\right)$ are sums of the eigenvalues of $\left(b_{j k}\right)$ taken $q$ at a time.

Lemma A.3. Let $\left(d_{j k}\right)$ be a Hermitian matrix and let $1 \leq q \leq n-2$. Then then $\left(\begin{array}{c}n-1 \\ q\end{array}\right)$ by $\left(\begin{array}{c}n-1 \\ q\end{array}\right)$ matrix $\left(D_{J J^{\prime}}^{q}\right)$ given by

$$
\begin{aligned}
& D_{J J}^{q}=\sum_{j \in J} b_{j j} \\
& D_{J J^{\prime}}^{q}=\sum_{\substack{1 \leq j, k \leq n-1 \\
j \neq k}} \epsilon_{j J^{\prime}}^{k J} b_{j k} \quad \text { if } J \neq J^{\prime},
\end{aligned}
$$

where $J$ and $J^{\prime}$ are multiindices, $|J|=\left|J^{\prime}\right|=q$ is also Hermitian. Moreover, the eigenvalues of $\left(D_{J J^{\prime}}^{q}\right)$ are sums of the eigenvalues of $\left(d_{j k}\right)$ taken $n-1-q$ at a time, so $\left(D_{J J^{\prime}}^{q}\right)$ is positive definite if $\left(d_{j k}\right)$ is positive definite and $n-1-q>0 ;\left(D_{J J^{\prime}}^{q}\right)$ is positive semi-definite if $\left(d_{j k}\right)$ is positive semi-definite for any $n$.

If $q=1$, then Lemma A.3 says that if $n \geq 3$ and $H=\left(h_{j k}\right)$ is a Hermitian, positive definite matrix, $1 \leq i, k \leq n-1$, then $\left(\delta_{j k} \sum_{\ell=1}^{n-1} h_{\ell \ell}-h_{j k}\right)$ is a Hermitian, positive definite matrix. The requirement that $n \geq 3$ is the seemingly technical reason that Theorem 1.1 is stated for $2 n-1 \geq 5$, as well as the results in [Nic06] and the fact that the work by Kohn and Nicoara in [KN06] assumes closed range of $\bar{\partial}_{b}$.

\section{REFERENCES}

[BS86] H. Boas and M. Shaw. Sobolev estimates for the Lewy operator on weakly pseudoconvex boundaries. Math. Ann., 274:221-231, 1986.

[BS91] H. Boas and E. Straube. Sobolev estimates for the complex Green operator on a class of weakly pseudoconvex boundaries. Comm. Partial Differential Equations, 16:1573-1582, 1991.

[Cat83] D. Catlin. Necessary conditions for subellipticity of the $\bar{\partial}$-Neumann problem. Ann. of Math., 117:147-171, 1983.

[Cat84] D. Catlin. Global regularity of the $\bar{\partial}$-Neumann problem. In Complex analysis of several variables (Madison, Wis., 1982), Proc. Sympos. Pure Math., 41, pages 39-49. Amer. Math. Soc., Providence, RI, 1984.

[Cat87] D. Catlin. Subelliptic estimates for the $\bar{\partial}$-Neumann problem on pseudoconvex domains. Ann. of Math., 126:131-191, 1987.

[D'A02] John P. D'Angelo. Inequalities from Complex Analysis. Number 28 in The Carus Mathematical Monographs. The Mathematical Association of America, Washingon, D.C., 2002. 
[Dia86] R. Diaz. Necessary conditions for subellipticity of $\square_{b}$ on pseudoconvex domains. Comm. in Partial Differential Equations, 11(1):1-61, 1986.

[FK72] G.B. Folland and J.J. Kohn. The Neumann problem for the Cauchy-Riemann Complex, volume 75 of Ann. of Math. Stud. Princeton University Press, Princeton, New Jersey, 1972.

[FS98] S. Fu and E. Straube. Compactness of the $\bar{\partial}$-Neumann problem on convex domains. J. Funct. Anal., 159(2):629-641, 1998.

[FS01] S. Fu and E. Straube. Compactness in the $\bar{\partial}$-Neumann problem. In Complex analysis and geometry (Columbus, OH, 1999), Ohio State Univ. Math. Res. Inst. Publ., 9, pages 141-160. de Gruyter, Berlin, 2001.

[Hör65] L. Hörmander. $L^{2}$ estimates and existence theorems for the $\bar{\partial}$ operator. Acta Math., 113:89-152, 1965.

[KN65] J.J. Kohn and L. Nirenberg. Non-coercive boundary value problems. Comm. Pure Appl. Math., 18:443-492, 1965.

[KN06] J.J. Kohn and A. Nicoara. The $\bar{\partial}_{b}$-equation on weakly pseudo-convex CR manifolds of dimension 3. J. Funct. Anal., 230:251-272, 2006.

[Koe02] K. Koenig. On maximal Sobolev and Hölder estimates for the tangential Cauchy-Riemann operator and boundary Laplacian. Am. J. Math, 124:129-197, 2002.

[Koe04] K. Koenig. A parametrix for the $\bar{\partial}$-Neumann problem on pseudoconvex domains of finite type. $J$. Funct. Anal., 216(1):243-302, 2004.

[Koh81] J.J. Kohn. Boundary regularity of $\bar{\partial}$. In Recent developments in several complex variables (Proc. Conf., Princeton Univ., Princeton, N.J., 1979), volume 100 of Ann. of Math. Stud., pages 243-260. Princeton Univ. Press, Princeton, N.J., 1981.

[Koh86] J.J. Kohn. The range of the tangential Cauchy-Riemann operator. Duke Math. J., 53:525-545, 1986.

[Koh02] J.J. Kohn. Superlogarithmic estimates on pseudoconvex domains and CR manifolds. Ann. of Math., 156:213-248, 2002.

[LN66] P. Lax and L. Nirenberg. On stability for difference schemes: a sharp form of Gårding's inequality. Comm. Pure Appl. Math., 19:473-492, 1966.

[Nic] Andreea Nicoara. Equivalence of types and Catlin boundary systems. arXiv:0711.0429.

[Nic06] A. Nicoara. Global regularity for $\bar{\partial}_{b}$ on weakly pseudoconvex CR manifolds. Adv. Math., 199:356447, 2006.

[RS08] Andrew S. Raich and Emil J. Straube. Compactness of the complex Green operator. Math. Res. Lett., 15(4):761-778, 2008.

[Sha85a] Mei-Chi Shaw. Global solvability and regularity for $\bar{\partial}$ on an annulus between two wekly pseudoconvex domains. Trans. Amer. Math. Soc., 291:255-267, 1985.

[Sha85b] Mei-Chi Shaw. $L^{2}$-estimates and existence theorems for the tangential Cauchy-Riemann complex. Invent. Math., 82:133-150, 1985.

[Str] Emil J. Straube. Lectures on the $\mathcal{L}^{2}$-Sobolev Theory of the $\bar{\partial}$-Neumann Problem.

[Str97] E. Straube. Plurisubharmonic functions and subellipticity of the $\bar{\partial}$-Neumann problem on nonsmooth domains. Math. Res. Lett., 4:459-467, 1997.

[Str06] E. Straube. Aspects of the $L^{2}$-Sobolev theory of the $\bar{\partial}$-Neumann problem. In Proceedings of the International Congress of Mathematicians, Madrid 2006, vol. II, pages 1453-1478. Eur. Math. Soc., 2006.

Department of Mathematical Sciences, 1 University of Arkansas, SCEn 327, FayetTEVILLE, AR 72701

E-mail address: araich@uark.edu 